Part A

\title{
3D Morphometry Aids Facial Analysis of Individuals with a Childhood Cancer
}

\begin{tabular}{|r|l|}
\hline Journal: & American Journal of Medical Genetics: Part A \\
\hline Manuscript ID & $15-0507 . R 2$ \\
\hline Wiley - Manuscript type: & Original Article \\
\hline Date Submitted by the Author: & n/a \\
\hline Complete List of Authors: & $\begin{array}{l}\text { Hopman, Saskia; Emma Children\'s Hospital/Amsterdam Medical Center, } \\
\text { Department of Pediatric Oncology } \\
\text { Merks, Johannes; Emma Children\'s Hospital/Amsterdam Medical Center, } \\
\text { Department of Pediatric Oncology } \\
\text { Suttie, Michael; University College London, Institute of Child Health } \\
\text { Hennekam, Raoul; Department of Pediatrics, Academic Medical Center } \\
\text { Hammond, Peter; University College London, ; }\end{array}$ \\
\hline Keywords: & $\begin{array}{l}\text { Three-dimensional imaging, 3D, morphometric analysis, morphological } \\
\text { abnormalities, face shape differences, childhood cancer }\end{array}$ \\
\hline Search Terms: & $\begin{array}{l}\text { Three-dimensional imaging, 3D, morphometric analysis, morphological } \\
\text { abnormalities, face shape differences, childhood cancer }\end{array}$ \\
\hline \multicolumn{2}{|c|}{} \\
\hline
\end{tabular}

\section{SCHOLARONE ${ }^{\text {'T }}$}

Manuscripts 


\title{
3D Morphometry Aids Facial Analysis of Individuals with a Childhood Cancer
}

\author{
Saskia M. J. Hopman ${ }^{1}$, Johannes H. M. Merks ${ }^{1}$, Michael Suttie ${ }^{2}$, Raoul C. M. Hennekam ${ }^{3}$, \\ Peter Hammond ${ }^{2}$ \\ 1: Department of Pediatric Oncology, Emma Children's Hospital, Academic Medical \\ Center, Amsterdam, Netherlands \\ 2: $\quad$ Genetics \& Genomic Medicine, UCL Institute of Child Health, London, UK \\ 3: Departments of Pediatrics and Clinical Genetics, Emma Children's Hospital, Academic \\ Medical Center, Amsterdam, Netherlands
}

Running title: 3D Analysis in Childhood Cancer Patients

\section{Corresponding author:}

Peter Hammond, Professor of Computational Biology

Genetics \& Genomic Medicine, UCL Institute of Child Health

30 Guilford St, London WC1N 1EH

UNITED KINGDOM

Phone: +44 (0)207 9052399

Fax : +44 (0)207404 6191

Email:p.hammond@ucl.ac.uk

John Wiley \& Sons, Inc. 


\begin{abstract}
A group of patients who had cancer as a child were previously found to have distinct patterns of morphological abnormalities. In this study, we investigated the added value of 3D shape analysis to characterize their facial morphology.

Primarily, we showed in an objective and quantitative manner that the overall facial dysmorphism of the individuals who had had a childhood cancer was significantly greater than that of the controls. We also demonstrated how the same approach can be used to detect a similar disparity for a more localized malar region comprising customized disconnected patches defined on both sides of the face. In addition, by comparing original face surfaces to their mirrored forms, we confirmed that the patient group had significantly greater facial asymmetry than the controls. Each of these results made use of surface shape differences not detectable by simple linear or angular characteristics as might be used in analyses based on measures captured manually or derived from landmarks annotating 2D photographic images.

We conclude that 3D morphometric analysis of a relatively small heterogeneous patient group can further delineate face shape differences from typically developing individuals that are too subtle or geometrically complex to identify or quantify objectively with conventional clinical and anthropometric approaches.
\end{abstract}


1

2

3

4

5

6

7

8

9

10

11

12

13

14

15

16

17

18

19

20

21

22

23

24

25

26

27

28

29

30

31

32

33

34

35

36

37

38

39

40

41

42

43

44

45

46

47

48

49

50

51

52

53

54

55

56

57

58

59

60

\section{KEYWORDS}

Three-dimensional imaging; 3D; morphometric analysis; morphological abnormalities; face shape differences; childhood cancer

John Wiley \& Sons, Inc. 


\section{INTRODUCTION}

Many genetic syndromes involve facial morphological characteristics, and the facial 'Gestalt' can be an important clue in the identification of genetic conditions [Hammond, 2007; Hammond et al., 2012b; Hennekam et al., 2010]. We also know that in tumor predisposition syndromes, the constitutional molecular defects that lead to oncogenesis may also play an important role in facial development [Ponder, 2001]. Traditionally, anthropometry has been an important tool in analyzing the phenotype of an individual [Farkas, 1994]. It has the disadvantage of inter-observer variability and requires prolonged co-operation of the subject as well as their presence for capturing additional measurements. This limits the use of anthropometry on children or individuals with an intellectual disability. Two-dimensional photography overcomes some of these limitations, but the quality and usability of a 2D picture is highly dependent on the experience of the photographer and cooperation, pose and lighting of the subject [Hammond, 2007].

Three-dimensional (3D) photogrammetric images are independent of pose, and offer repeat measurement without the subject's presence or prolonged cooperation [Hammond et al., 2012a]. Typically, 3D face images are annotated with anatomical landmarks to produce linear and angular measurements. For facial analysis, dense surface modelling (DSM) techniques induce tens of thousands of densely corresponded points as quasilandmarks from a small number of manually placed landmarks. Using DSMs, the average surface of a group of faces can be computed and quantitative shape comparisons of face surfaces can be performed between individuals or relative to ethnically, age- and sexmatched means [Hammond \& Suttie, 2012a]. DSM based analysis has successfully delineated the facial phenotype of a variety of neurodevelopmental conditions [Hammond 
et al., 2005; Hammond et al., 2008].

In a previous study, we used 'traditional' anthropometry and morphological examination in a cohort of 1,073 individuals who had cancer as a child, and compared these to 1,007 healthy schoolchildren [Merks et al., 2005]. We demonstrated significantly increased incidence of morphological abnormalities and high prevalence of syndromes. Furthermore, we described four new patterns of co-occurring morphological abnormalities as tumor predisposition syndromes [Merks et al., 2008] within this cohort. These were named after key abnormalities: "blepharophimosis (BP) pattern", "epicanthal folds (EF) pattern", "asymmetric lower limbs (LLA) pattern" and "Sydney creases (SC) pattern" (Table 1). The structural genomic variants identified in patients showing one of the four patterns were reported recently [Hopman et al., 2013a]. The goal of the present study is to evaluate whether using 3D shape analysis in this same subgroup of childhood cancer patients adds to the evaluation of facial morphology by clinical inspection. More specifically, to determine objectively if there is a difference in dysmorphisms observed between childhood cancer patients and healthy controls. Additional objectives are to objectively determine the shape of a geometrically complex facial region, i.e. the malar region, that previously could be evaluated only subjectively, and to identify additional morphological signs within the patient group that were previously undetected by clinical inspection. We hypothesize that childhood cancer patients show more dysmorphisms compared to healthy controls (regardless effects of therapy modalities). 


\section{SUBJECTS AND METHODS}

\section{Patients and control subjects}

In an earlier study we detected differences in face-shape between Dutch individuals and individuals from the UK, phylogenetically-related populations [Hopman et al., 2013b]. Therefore, in current studywe confined our analyses to subjects of self-reported Dutch descent. Forty-nine childhood cancer patients, previously designated as showing one of four patterns of morphological abnormalities [Merks et al., 2008; Hopman et al., 2013a] were asked to participate and only one declined. In this cohort, there were no patients who had undergone local therapy in the facial region and all patients had finished their cancer therapy for more than five years. The 3D photos of 7/48 patients were excluded for technical reasons or due to their non-Dutch descent [Hopman et al., 2013b]. The characteristics of the patient subgroups are shown in Table II.

The control group consisted of 274 subjects of self-reported Dutch descent recruited from university scientific and hospital medical professionals $(81 / 274,30 \%)$ and unaffected parents and sibs of children with a molecularly proven genetic syndrome who visited outpatient departments or attended family support group meetings (193/274, 70\%). The composition and average ages of patient and control groups are shown in Table III.

\section{Image capture and preparation}

3D facial imaging was undertaken using commercial stereo-photogrammetric cameras able to capture more than 25,000 3D points on an adult face surface (Canfield Imaging Systems, New Jersey, USA; 3dMD, Atlanta, USA). Each face image was manually annotated using inhouse software with 22 landmarks previously shown to be reliable and reproducible 
[Gwilliam et al., 2006]. The landmarks were used to induce a dense correspondence across all subjects of all $25,000+$ surface points. A DSM of all faces in the dataset was generated as the set of principal component analysis (PCA) modes covering $99 \%$ of shape variation of face shape difference from the overall mean face. DSM construction involved methods developed in-house which are described elsewhere [Hutton, 2004; Hammond et al., 2005; Hammond \& Suttie, 2012a].

Subjective morphological signs that show differences in a specific direction were evaluated along an appropriate axis (lateral, vertical, anterior-posterior) and in a model confined to a region containing the sign under study [Hammond \& Suttie, 2012a]. Separate DSMs of the reduced face without ears and the malar region (Figure 2A) were computed. Ears were omitted because of their frequent partial, or occasional complete, occlusion by hair.

\section{Statistical analyses}

For the continuous outcome variables with a normal distribution, student-t-tests were performed using a significance level of 0.05 . For t-tests, only the controls in the patient defined age range of 10 to 40 years were included. Anthropometric calculations and t-tests were performed in Excel (Microsoft Office 2010) and StatPlus (AnalystSoft, version 5.7.5). In order to maximize the analysis of the relatively small number of female patients, male and female asymmetry measures were adjusted for sex and age based on the control population before combined analysis. Age adjustment was achieved using separate age-based linear regressions for male and female controls, and the formula:

$$
\text { adjusted }=\left(\text { orginal }-m^{*} \text { age }-c\right) / \text { sqrt }\left(1+m^{*} m\right)
$$


where $y=m * x+c$ is the equation of the regression line for original against age. Following age adjustment, z-scores were computed based on means and standard deviations for the appropriate age range and same sex controls.

Bootstrap analyses were performed to sample estimates of the difference in the mean asymmetry indices, mean overall facial dysmorphism scores and mean dysmorphism score for the malar region respectively. We compared the means of for each of these values for patients and controls with 1000 bootstrap samples. The overall facial dysmorphism scores dysmorphism scores for the malar region and asymmetry indices were adjusted for sex, age and ethnicity. As a measurement of effect size, we calculated the Cohen's D value as the difference between the means divided by the pooled SD for overall facial dysmorphism scores dysmorphism scores for the malar region and asymmetry indices.

\section{Face signatures and colour-coded heat maps of average and individual faces}

Each individual was matched with 50 age-contiguous same sex controls whose mean age most closely matched that of the individual. The displacements of the densely corresponded points on an individual's face were normalized with respect to corresponding displacements on the faces of matched controls to produce a face signature [Hammond et al., 2012b]. In order to match the age range of the patient group, only control subjects aged between 10 and 40 years were included in signature graph analysis (the full age range was employed when selecting age-sex matched controls).

Individual face signatures were heat mapped to visualize normalized shape differences from matched controls using a red-green-blue colour scale at min-0-max units of standard deviation for appropriate minima and maxima (-2SD and +2SD unless otherwise stated). Thus, for differences normal to the face surface, the red/green/blue spectrum 
corresponded to contraction/coincidence/expansion of the surface being compared. Analogous processes produce signatures for surface shape differences parallel to lateral, vertical, and depth (anterior-posterior) axes. The signature weight of an individual face or face patch (square root of the sum of the squared normalized differences for all densely corresponded points) reflects the deviation of a subject from matched controls and thus defines a useful estimate of the facial dysmorphism of an individual.

A face signature graph is constructed using face signatures as vertices or nodes and a directed edge links each vertex to its most "similar" signature. An alternative, but topologically identical, "coloured" form of a face signature paints the vertices to reflect a diagnostic or other subgroup labelling such as control-patient dichotomy or tumor disposition pattern. Finally, a "coloured" signature graph can be "collapsed" to a simplified form where vertices represent clusters of same-coloured connected vertices of the original [Hammond \& Suttie, 2012a]. An entropy-like measure is used to summarize the associated clustering or dispersion of the members of each labelled subgroup. The lower the dispersion factor of a labelled subgroup, the stronger is the similarity of the facial dysmorphism of subcluster members compared to other subgroups. A maximum dispersion factor of 1.00 means that a subgroup's members are perfectly dispersed into singleton subsets and so do not cluster at all because they lack homogeneity and differ from other labelled subgroups in their dysmorphism. All signature graphs were drawn using the open source software GraphViz (version 2.26, www.graphviz.org).

\section{Facial asymmetry}

As in previous studies of facial asymmetry, we constructed a DSM containing the original and reflected (mirrored form) of the face surfaces of all study participants. The manually 
placed and left-right-paired landmarks were swapped over before resampling the surface, effectively interpolating a dense set of landmarks on both sides of the face. Thus, the interpolated landmarks are matched-paired across the face; to study face asymmetry, a DSM was constructed from the combined set of densely corresponded face surfaces and their mirrored forms. We estimated individual facial asymmetry as the Euclidean distance between the DSM representations of the original and mirrored face surfaces. [Hammond et al., 2008].

This was adjusted to take age and sex into consideration since there are gender differences and asymmetry tends to increase with age within the general population [Hammond et al., 2008; Claes et al., 2012a].

\section{RESULTS}

\section{The individuals with a childhood cancer have greater facial dysmorphism than age-sex-} ethnicity matched controls but no set of common dysmorphic features, not even within the tumor predisposition patterns found previously

The heat mapped signatures in Figure $1 A$ delineate localized individual facial dysmorphism of 12 female (row 1) and 29 male patients of Dutch descent (rows 2 to 4 ). This visualization of detailed face shape differences offers an objective adjunct to clinical examination. It is possible to identify small subgroups of patients with a similar heat map highlighting similar facial dysmorphism such as malar flattening (anterior red patches on cheeks of LLA_9, LLA_24, LLA_34, LLA_43), perioral insufficiency (red regions around mouths of LLA_29, LLA_45, SC_3, SC_26, LLA_29) and mid-facial hyperplasia (blue patches on midface of EF_2, EF_7, LLA_20). However, overall, there is considerable heterogeneity in the facial dysmorphism of the patient group. This is confirmed in the collapsed form of the face 
signature graph for controls and patients (Figure 1C) with dispersion factors of 0.94 for patients and 0.16 for controls, and the patient group's clustering as a large number of singletons, a few duos and a quartet.

The location of patients in the periphery or as terminal nodes of the signature graph for controls and patients is as a result of their greater facial dysmorphism (Figure 1B).

Furthermore, the associated (log transformed) face signature weight distribution (Figure 1D) confirms a significantly greater mean for patients $(M=2.20)$ compared to controls $(M=2.14)$; $t(54)=2.35, p=0.02$

The bootstrap analysis shows a difference of $0.67(95 \% \mathrm{Cl} 0.014-0.124, \mathrm{p}<0.05)$ between the mean overall facial dysmorphism score for patients and controls. The Cohen's d value for effect size of the overall facial dysmorphism score (adjusted for sex, age and ethnicity) between patients and controls is 0.4 (i.e. between medium and large effect size). Inspection of the face signatures in Figure $1 A$ suggests the LLA pattern subgroup to have more red-blue hues than green and hence to be more facially dysmorphic. Some quantitative evidence for this is seen in a control vs pattern histogram of signature weights (Supplementary Figure 1) but the relatively low numbers in the other pattern subgroups rather undermines such cross pattern comparisons.

\section{The malar region is more dysmorphic in patients compared to controls}

Although, as shown above, there was no discernible set of common dysmorphic features in the patient group when considering the face in its entirety, the malar region is notably painted red or blue (at 2SD) in a majority of the heat mapped signatures of Figure $1 \mathrm{~A}$. This clinical observation suggested that the malar region should undergo closer objective 
scrutiny. Given the complex geometry of the malar region, this would be impossible using linear or angular measures derived solely from landmarks. In order to investigate malar dysmorphism, a customized region was demarcated as depicted in Figure $2 \mathrm{~A}$.

The distribution of signature weight as a measure of dysmorphism for the malar region is shown in the histogram in Figure $2 B$. The malar dysmorphism score is indeed significantly higher in patients $(M=1.81)$ compared to controls $(M=1.74) ; t(58)=2.93, p=0.004$. The bootstrap analysis shows a difference of $0.52(95 \% \mathrm{Cl} 0.019-0.127, \mathrm{p}<0.05)$ between the mean dysmorphism score of the malar region for patients and controls. The Cohen's $d$ value for effect size of the dysmorphism score for the malar region (adjusted for sex, age and ethnicity) between patients and controls is 0.5 (i.e. between medium and large effect size).

The collapsed signature graph for the malar region for controls and patients, as was the case for the face, shows patients to be located more at the periphery, reflecting greater malar dysmorphism. The malar region dispersion factors for controls $(0.16)$ and patients (0.92) also reflect the heterogeneity of the malar dysmorphism for the patients when considered as a whole (Supplementary Figure 2).

Interestingly, the signature graph for differences along the anterior-posterior or zaxis of the malar region for patients alone shows some stronger clustering for LLA patients compared to other pattern subgroups (Figure $2 C$ ). This is more clearly shown in the collapsed form of the signature graph and the lower dispersion factor (0.66) for LLA-pattern patients (Figure 2D). In comparison, EF-, SC- and BP-pattern patients show dispersion factors of $0.83,0.87$ and 1.00 respectively. This suggests that the LLA-pattern subgroup of these 41 patients are more similar in their malar flattening or malar prominence than patients from the other pattern subgroups (Figure 2D). 


\section{Patients have significantly greater facial asymmetry when compared to controls}

The PCA-vector representation of each original face surface was subtracted from that for its mirrored form in the DSM generated for all original and mirrored or reflected images. The "size" of this vector difference is an estimate, or index, of the overall asymmetry of the original face surface. Facial asymmetry increases with age and at different rates for males and females as is shown by the separate male and female scatter plots for the raw asymmetry index against age (Supplementary Figures 3A and 3B)[Hammond et al., 2008]. When adjusted for age (linear regression adjusted) and z-scored for gender within the approximate patient age range of 10 to 40 years, there was significantly greater asymmetry in patients $(M=0.75)$ compared to controls $(M=0 ; t(52)=2.2, p=0.03)$. The bootstrap analysis shows a difference of $0.8006(95 \% \mathrm{Cl} 0.17-1.57, \mathrm{p}<0.05)$ between the mean adjusted asymmetry indices for patients and controls. The Cohen's d value for effect size of the asymmetry index (adjusted for sex, age and ethnicity) between patients and controls is 0.4 (i.e. between medium and large effect size).

This greater facial asymmetry for patients compared to controls can also be appreciated on an individual basis by interpreting the PCA vector difference between original and mirrored DSM PCA vectors as a surface in the DSM model. The resulting original-reflected difference for each patient can then be normalized against corresponding control differences just as for signatures for the original faces. Thus, corresponding to the gallery of face signatures of Figure 1 is another showing normalized asymmetry differences from the asymmetry of age-sex matched controls in the form of heat mapped facial asymmetry signatures (Figure $3 A$ ). Once again, it is possible to discern subgroups, this time with similar asymmetry dysmorphism. This is more easily observed by considering the 
induced clustering in the asymmetry signature graph of the patients alone (Figure 3B). Of the seven subclusters within the graph, subcluster 2 contains a duo of faces showing almost no difference (mostly green hue) from the asymmetry of age-sex-ethnicity matched controls. Subcluster 3 contains 8 individuals displaying a right dominant asymmetry difference from controls in the supraorbital region (blue above right eye, red above left eye). Indeed, the faces in subclusters 3, 4 and 5, below and to the left of subcluster 2, largely show right dominant asymmetry differences from controls, whereas subclusters 1, 6 and 7, above and to the right of subcluster 2, largely include individuals with more left dominant asymmetry. It also noticeable that most mandibular asymmetry occurs in the EF pattern. However, the small sizes of the pattern subgroups in this patient population somewhat undermines such attempts to hypothesize about intra-pattern similarities and inter-pattern differences.

\section{DISCUSSION}

The main purpose of this study was to identify additional benefits that 3D facial morphometry might offer beyond the clinical and classical anthropometric facial analysis carried out in the origin Merks et al study [Merks et al., 2005] of more than 1,000 individuals who had suffered a childhood cancer and a similarly sized group of controls. In the previous study, tumor predisposing syndrome patterns were identified by considering not just the face but other physical attributes as well. Inevitably, in this study of only faces, and in less than $3 \%$ of the original patient cohort, it was unlikely that the original patterns could be confirmed or that novel patterns might emerge. With the future study in mind of tens of thousands of individuals who have had a childhood cancer, it was more important that this study determine how best to exploit 3D facial morphometric analysis in the future 
on a much larger scale and in a much more heterogeneous population than has generally been undertaken hitherto.

Rather than investigate individual linear or angular measures derived from landmarks, we employed signature based analysis where surface shape differences at $25,000+$ surface points are normalized against age-sex-ethnicity matched controls for the whole face, for face patches and for the difference between an original face and its mirrored or reflected form. We used heat maps of the signatures to delineate individual localized dysmorphism. The "weight" of the signatures was used to estimate an index of dysmorphism, through which we established statistically significant differences between the patients and controls within the same age range. Signature graphs with directed edges linking most similar signatures were used to cluster both controls and patients into subsets with similar dysmorphism i.e. shape differences from age-sex matched controls. Relative central or peripheral position in signature graphs reflected low or high levels of dysmorphism and, in the collapsed form of signature graphs, rates of dispersion quantified overall clustering rates of patients compared to controls and/or between pattern subgroups of patients.

Using the methodological approach summarized above, we detected significantly higher dysmorphism scores (signature weights) for the facial region without ears in the individuals who had cancer as a child. This was also reflected in the corresponding signature graph for controls and patients where the latter patients appeared more in the periphery and as terminal nodes. Common facial differences between patients and controls could not be identified and a high dispersal rate, reflecting lack of similarity clustering of the patient group, provided supporting evidence. Similarly, when considering patients on their own, no intra-pattern similarities or inter-pattern differences were evident in the signatures or the signature graphs. Based on subjective evaluation of the face signatures, we hypothesized 
that the malar region of patients was more dysmorphic than in controls. This would be a challenging hypothesis to test using linear or angular measures derived from clinical examination or derived from landmarked photographs. It required an analysis of 3D surface shape. An experienced dysmorphologist $(\mathrm{RCMH})$ defined a customized region to act as the focus for analyzing shape differences in the malar region and as a base shape for constructing DSMs. Following the same methodological sequence as for face shape analysis, we established significantly greater dysmorphism of the malar region in the patients compared to controls. Once again, shape signature graphs were used to confirm this. In terms of intra-pattern analysis of anterior-posterior malar shape difference, the LLA pattern subgroup produced a lower dispersion rate suggesting some commonality of form for malar flattening or prominence. We should realize that typically in evolutionary, developmental and systematic biology, we use geometric morphometrics to measure form and its variations. These approaches are often unsuitable for describing phenotypes expressing discordant shape variation that do not occur in the normal population at all, not even under extreme forms. Ultimately, it requires extension to geometric morphometrics to quantify abnormal shape variation and with that, a redefinition of what is called dysmorphism. Claes et al. demonstrate the use of so called dysmorphometrics to measure unusual form differences given only normative data. It builds upon existing geometric morphometric techniques, but models form differences explicitly as outliers. This could have important implications for biomedical practice and research [Claes et al., 2012b].

Finally, we identified increased facial asymmetry in patients compared to controls. This could have been an effect of therapy that childhood cancer patients may have received. However, patients who had undergone radiotherapy in the facial region were excluded. Therefore, the increased facial asymmetry in patients is more likely to reflect 'intrinsic 
asymmetry' rather than asymmetry caused by external effects. We should note that the asymmetry analyses we performed in this paper do not include a test for significance of asymmetry over measurement error. Previous studies have considered fluctuating asymmetry: nondirectional deviations from bilateral symmetry [Palmer and Strobeck 1986], including a procedure to make bilateral variation serve as a measurement of developmental precision [Palmer and Strobeck 1997]. This was explored further in terms of the heritability of fluctuating asymmetry [Klingenberg et al., 1999] and on shape analysis of symmetric structures to quantify asymmetry (and variation) among individuals [Klingenberg et al., 2002]. It is recommended that in asymmetry analyses, tests for significance of asymmetry (above measurement error), directional asymmetry (side-bias) and deviation from normality (antisymmetry) are incorporated. They propose a framework to do so [Klingenberg et al., 2002; Klingenberg 2015] for asymmetry analyses of paired simple measures or for tens of left-right paired landmarks. The 3D asymmetry estimate in our study is based on PCA mode scores for 3D surfaces comprising $25,000+$ unpaired surface points. At this point, it is unclear to us how the proposed methodology might be applied to our situation. This is an important omission to be addressed in the literature.

Asymmetry occurs frequently in genetic conditions associated with a malignancy. For example, it is a well-known feature in Bannayan-Riley-Ruvalcaba syndrome (BRRS, OMIM: 153480) [Bannayan, 1971] and Beckwith-Wiedemann Syndrome (BWS, OMIM: 13060) [Wiedemannnn, 1964]. It should be noted that none of the patient subgroup of this study were diagnosed with established syndromes such as BRRS or BWS. Some potential for interand intra-pattern analysis of facial asymmetry was identified for particular parts of the face, e.g. supra-orbit and mandible, and may assist in future determination of face shape differences and even alternative tumor disposition patterns of dysmorphism. 
In this project, we used 3D facial photographs and dense surface modelling morphometric techniques to characterize the facial phenotype of a cohort of patients who had cancer as a child, and in whom distinct patterns of morphological abnormalities were previously demonstrated using physical examination and anthropometry [Merks et al., 2008]. The patterns consist of abnormalities located both in the face and in other body parts. As we used only the face, it is impossible to evaluate the complete morphology of patterns this way. Therefore, it was impossible to repeat the grouping of the patients using signature graphs for the complete cohort (1,073 childhood cancer patients) described by Merks et al. [Merks et al., 2008], blinded from the statistical patterns of co-occurring morphological abnormalities. The relatively small number of individuals of the various patterns and in the total cohort hampers detailed statistical analyses and prevents some subgroup analysis. Another overall limitation of our analysis could be that, in computing face signatures, the age- and sex-matched controls selected are according to a matching running mean age, which could be biased by lack of coverage of certain ages.

Despite these limitations, the 3D face image capture and subsequent analysis has shown significant added value. We conclude that dense surface modelling techniques expand the possibilities for physicians (plastic surgeons, clinical geneticists etc.) to describe and characterize the phenotype of an individual or group of individuals and allow objective comparisons to age- and sex-matched controls. The addition of the approach adopted here in a large, nation-wide study of individuals who have had cancer as a child, together with a recently developed tool to recognize individuals with a high chance to have a tumor predisposition syndrome [Hopman et al., 2013c] will allow recognition of larger subgroups within pediatric cancer patients. Signature graphs can help pre-sorting the patients into clusters with similar differences from the control group and allow further characterization of 
the faces and facilitate subsequent molecular analyses. Such a study is presently in preparation.

\section{ACKNOWLEDGEMENTS}

The authors would like to thank all participants of the study. PH and MS received part funding from the US National Institute on Alcohol and Alcohol Abuse (NIAAA) through a CIFASD developmental grant (www.CIFASD.org). SMJH was supported by the Tom Voûte Fund. 
SEPARATE BOX

\section{DEFINITIONS AND EXPLANATION OF FREQUENT USED TERMS ${ }^{a}$}

LANDMARKS: Designated soft tissue points on anatomically well-defined locations.

EUCLIDEAN DISTANCE: Mathematic term for distance between two n-dimensional vectors defined as square root of sum of $n$ squared component-wise differences.

DENSE SURFACE MODEL (DSM): The actual 3D model, which forms the basis for further 3D analysis. A DSM is built from a set of surfaces of which mean landmarks are calculated using the Procrustes algorithm. The surfaces of the individual subjects are warped to the mean landmarks, aligning them. The points on a selected face can be mapped to the closest points on each face to induce a dense correspondence of tens of thousands of images across all images. The DSM is formed from the principal components covering $99 \%$ of shape variation from the overall average based on the densely corresponded points.

FACE SIGNATURE: The normalized differences, typically orthogonal to the face surface, of surface point displacements from corresponding positions of the densely corresponded points on the mean surface.

SIGNATURE WEIGHT: The square root of the sum of the squared normalized differences for all densely corresponded points.

DYSMORPHISM SCORE: The deviation of a subject from matched controls calculated by the signature weight of an individual face or face patch (square root of the sum of the squared normalized differences for all densely corresponded points). 
FACE SIGNATURE DISTANCE (FSD): An automated partial ordering of face signatures is defined as the Euclidean distance between the vectors representing the normalized differences across the densely corresponded points. A face signature graph can then be constructed with a set of face signatures as its vertices. A directed edge is drawn from each signature to another, possibly not unique, signature with the smallest FSD from the first,or equivalently the most similar dysmorphism to the first. The length of an edge between two vertices is the FSD between them. The shorter the edge, the more similar is the nature of the facial dysmorphism of the linked faces.

SIGNATURE GRAPH: Graphical representation of a set of face signatures as vertices. A directed edge is drawn from one signature to another signature with the smallest FSD from the first. In a signature graph, the FSD is the length of the edge between two vertices.

LINEAR MEASUREMENTS: Calculations of anthropometric distances using the 3D coordinated of anatomical landmarks.

${ }^{\text {a }}$ Adapted from [Hammond \& Suttie, 2012a] and [Hammond et al., 2012b] 
Allanson JE, Biesecker LG, Carey JC, Hennekam RC. 2009a. Elements of morphology: introduction. Am.J.Med.Genet.A 149A: 2-5.

Allanson JE, Cunniff C, Hoyme HE, McGaughran J, Muenke M, Neri G. 2009b. Elements of morphology: standard terminology for the head and face. Am.J.Med.Genet.A 149A: 6-28.

Bannayan GA. 1971. Lipomatosis, angiomatosis, and macrencephalia. A previously undescribed congenital syndrome. Arch.Pathol. 92: 1-5.

Biesecker LG, Aase JM, Clericuzio C, Gurrieri F, Temple IK, Toriello H. 2009. Elements of morphology: standard terminology for the hands and feet. Am.J.Med.Genet.A 149A: 93-127.

Carey JC, Cohen MM, Jr., Curry CJ, Devriendt K, Holmes LB, Verloes A. 2009. Elements of morphology: standard terminology for the lips, mouth, and oral region. Am.J.Med.Genet.A 149A: 77-92.

Claes P, Walters M, Shriver MD, Puts D, Gibson G, Clement J, Baynam G, Verbeke G, Vandermeulen D, Suetens P. 2012a. Sexual dimorphism in multiple aspects of 3D facial symmetry and asymmetry defined by spatially dense geometric morphometrics. J.Anat. 221: 97-114.

Claes P, Daniels K, Walters M, Clement J, Vandermeulen D, Suetens P. 2012b.

Dysmorphometrics: the modelling of morphological abnormalities. Theoretical Biology and Medical Modelling. 9: 5

Farkas L. 1994. New York: Raven Press. Anthropometry of the head and face in medicine. 
Gwilliam JR, Cunningham SJ, Hutton T. 2006. Reproducibility of soft tissue landmarks on three-dimensional facial scans. Eur.J.Orthod. 28: 408-415.

Hall BD, Graham JM, Jr., Cassidy SB, Opitz JM. 2009. Elements of morphology: standard terminology for the periorbital region. Am.J.Med.Genet.A 149A: 29-39.

Hammond P. 2007. The use of 3D face shape modelling in dysmorphology. Arch.Dis.Child 92: $1120-1126$.

Hammond P, Forster-Gibson C, Chudley AE, Allanson JE, Hutton TJ, Farrell SA, McKenzie J, Holden JJ, Lewis ME. 2008. Face-brain asymmetry in autism spectrum disorders. Mol.Psychiatry 13: 614-623.

Hammond P, Hutton TJ, Allanson JE, Buxton B, Campbell LE, Clayton-Smith J, Donnai D, Karmiloff-Smith A, Metcalfe K, Murphy KC, Patton M, Pober B, Prescott K, Scambler P, Shaw A, Smith AC, Stevens AF, Temple IK, Hennekam R, Tassabehji M. 2005. Discriminating power of localized three-dimensional facial morphology. Am.J.Hum.Genet. 77: 999-1010.

Hammond P, Suttie M. 2012a. Large-scale objective phenotyping of 3D facial morphology. Hum.Mutat. 33: 817-825.

Hammond P, Suttie M, Hennekam RC, Allanson J, Shore EM, Kaplan FS. 2012b. The face signature of fibrodysplasia ossificans progressiva. Am.J.Med.Genet.A 158A: 1368-1380. Hennekam RC, Allanson JE, Biesecker LG, Carey JC, Opitz JM, Vilain E. 2013. Elements of morphology: Standard terminology for the external genitalia. Am.J.Med.Genet.A 161: 12381263. 
Hennekam RC, Cormier-Daire V, Hall JG, Mehes K, Patton M, Stevenson RE. 2009. Elements of morphology: standard terminology for the nose and philtrum. Am.J.Med.Genet.A 149A: 61-76. Hennekam RCM, Krantz ID, Allanson JE. 2010. Gorlin's syndromes of the head and neck. (5th ed.) New York, USA: Oxford University Press Inc.

Hopman S, Merks J, Eussen H, Douben H, Snijder S, Hennekam R, de KA, Caron H. 2013a. Structural genome variations in individuals with childhood cancer and tumour predisposition syndromes. Eur.J.Cancer. 4: 2170-8.

Hopman S, Merks J, Suttie M, Hennekam R, Hammond P. (2013b). Face shape differs in phylogenetically related populations. Eur J Hum Genet. 22: 1268-71Hopman SM, Merks JH, de Borgie CA, Aalfs CM, Biesecker LG, Cole T, Eng C, Legius E, Maher ER, van Noesel MM, Verloes A, Viskochil DH, Wagner A, Weksberg R, Caron HN, Hennekam RC. 2013c. The development of a clinical screening instrument for tumour predisposition syndromes in childhood cancer patients. Eur.J.Cancer. 49: 3247-54.

Hunter A, Frias JL, Gillessen-Kaesbach G, Hughes H, Jones KL, Wilson L. 2009. Elements of morphology: standard terminology for the ear. Am.J.Med.Genet.A 149A: 40-60.

Hutton TJ. (2004). Dense surface models of the human face. Thesis/dissertation University College London, London.

Klingenberg CP, Nijhout HF. 1999. Genetics of fluctuating asymmetry: a developmental model of developmental instability. Evolution. 53: 358-375.

Klingenberg CP, Barluenga M, Meyer A. 2002.Shape analysis of symmetric structures: quantifying variation among individuals and asymmetry. Evolution.56:1909-1920.

John Wiley \& Sons, Inc. 
Klingenberg CP. 2015. Analyzing fluctuating asymmetry with geometric morphometrics: concepts, methods and applications. Symmetry. 7: 843-934.

Merks JH, Caron HN, Hennekam RC. 2005. High incidence of malformation syndromes in a series of 1,073 children with cancer. Am.J.Med.Genet.A 134A: 132-143.

Merks JH, Ozgen HM, Koster J, Zwinderman AH, Caron HN, Hennekam RC. 2008. Prevalence and patterns of morphological abnormalities in patients with childhood cancer. JAMA 299: 61-69.

Palmer AR, Strobeck C. 1986. Fluctuating asymmetry: measurement, analysis and patterns. ann. Rev. Ecol. Syst. 17: 391-421.

Palmer AR, Strobeck C. 1997. Fluctuating asymmetry and developmental stability: heritability of observable variation vs. heritability of inferred cause. J. Evol. Biol. 10: 39-49. Ponder BA. 2001. Cancer genetics. Nature 411: 336-341.

Wiedemann HR. 1964. Familial malformation complex with umbilical hernia and macroglossia- a "new syndrome"? J.Genet.Hum. 13: 223-232.

John Wiley \& Sons, Inc. 


\section{TABLES}

\begin{tabular}{|c|c|}
\hline $\begin{array}{l}\text { Name pattern of co-occurring morphological } \\
\text { abnormalities }\end{array}$ & Morphological abnormalities \\
\hline $\begin{array}{l}\text { BLEPHAROPHIMOSIS PATTERN } \\
(\mathrm{n}=10)\end{array}$ & $\begin{array}{l}\geq 2 \text { of the following: } \\
\text { - Blepharophimosis } \\
\text { - Patchy hypopigmentation } \\
\text { - Café-au-lait spots } \\
\text { - Increased angulation of the spine }\end{array}$ \\
\hline $\begin{array}{l}\text { ASYMMETRIC LOWER LIMBS PATTERN } \\
(\mathrm{n}=17)\end{array}$ & $\begin{array}{l}\geq 2 \text { of the following: } \\
\text { - Asymmetric lower limbs } \\
\text { - Ptosis } \\
\text { - Malar flattening }{ }^{*} \\
\text { - Pectus carinatum or pectus excavatum }\end{array}$ \\
\hline $\begin{array}{l}\text { EPICANTHAL FOLDS PATTERN } \\
(\mathrm{n}=8)\end{array}$ & $\begin{array}{l}\geq 3 \text { of the following: } \\
\text { - Epicanthus }{ }^{*} \\
\text { - Flat wide nose, broad nasal tip, wide nasal } \\
\text { - Thick vermillion of the upper and/or } \\
\text { lower lip }{ }^{*} \\
\text { - Protruding ears }\end{array}$ \\
\hline
\end{tabular}


Table I: Summary of patterns of statistically significant co-occurring morphological abnormalities and number of patients in each pattern for 3D analyses. In the current study 41 patients were available for 3D facial analyses. The patients from the current cohort were selected from a large prospective cohort of childhood cancer patients and all showed one of the four patterns of statistically significant co-occurring morphological abnormalities. The table shows the criteria for each of the patterns and the number of patients included from each pattern, based on the morphological examination by Merks et al. (partly described in [Merks et al., 2008]). This table is adapted from [Hopman et al., 2013a].

*= Modified according to Elements of Morphology nomenclature [Allanson et al., 2009a; Hunter et al., 2009; Hennekam et al., 2013; Biesecker et al., 2009; Allanson et al., 2009b; Carey et al., 2009; Hennekam et al., 2009; Hall et al., 2009]. 


\begin{tabular}{|c|c|c|c|}
\hline SEX & PATTERN & SUBJECTS & MEAN AGE \\
\hline \multirow{4}{*}{$\begin{array}{l}\text { Male } \\
(\mathrm{N}=29)\end{array}$} & $L L A$ & 17 & 24.9 \\
\hline & $B P$ & 4 & 27.3 \\
\hline & $S C$ & 3 & 11.0 \\
\hline & $E F$ & 5 & 23.6 \\
\hline \multirow{4}{*}{$\begin{array}{l}\text { Female } \\
(\mathrm{N}=12)\end{array}$} & $L L A$ & 0 & 24.9 \\
\hline & $B P$ & 6 & 21.8 \\
\hline & $S C$ & 3 & 15.2 \\
\hline & $E F$ & 3 & 25.7 \\
\hline
\end{tabular}

Table II: Characteristics of patient subgroups

\begin{tabular}{|l|l|c|c|}
\hline \multicolumn{1}{|c|}{ SEX } & GROUP & SUBJECTS & MEAN AGE \\
\hline \multirow{2}{*}{ Male } & Patients & 29 & 23.6 \\
\cline { 2 - 4 } & & & \\
\hline \multirow{2}{*}{ Female } & Controls & 138 & 30.5 \\
\cline { 2 - 4 } & & 12 & 21.1 \\
\cline { 2 - 4 } & Controls & 136 & 28.0 \\
\hline
\end{tabular}

Table III: Characteristics of patient and control groups 


\title{
LEGENDS TO FIGURES
}

\section{Figure 1 Face signatures of patients; and signature weights and graphs for patients and controls.}

\begin{abstract}
A: Heat mapped face signatures of 41 patients of Dutch descent. Each demarcates localized facial dysmorphism using a red/green/blue scale for "below -2SD"/zero SD/"over +2SD" contractive/coincident/expansive surface difference compared to a mean of age-sexethnicity matched controls.

B: Face signature graph for patients (with square outline) and controls. Typically, in a signature graph the least dysmorphic individuals are located more centrally and the most dysmorphic more peripherally. The location of patient signatures largely at the periphery and terminally reflects their greater facial dysmorphism.

C. Collapsed version of the face signature graph for patients and controls, shown as empty and filled circles respectively. The annotated dispersion rates, between 0 and 1 , summarize the overall clustering of the control and patient signatures. The low value $(0.16)$ for controls and high value for patients $(0.94)$ suggests that the patient dysmorphism is significantly different from the majority of controls. The high value for the patient group also reflects the relatively large number of clusters into which it is decomposed and the lack of homogeneity in the facial dysmorphism of its members.

D: Histogram of log-transformed normalized signature weights showing greater overall facial dysmorphism in faces of patients compared to controls.
\end{abstract}

John Wiley \& Sons, Inc. 
Figure 2 Malar region definition, signature weight (for anterior-posterior depth axis)

A: A butterfly-shaped patch of the face surface was used to demarcate a malar region. The right inner canthus $\left(I C_{R}\right)$ was connected with the right exocanthus $\left(O C_{R}\right)$ following the lower orbit border. The right exocanthus was connected with the right lower ear attachment $($ LEA $)$ and the upper ear attachment $\left(U E A_{R}\right)$ was connected with the right cheilion $\left(\mathrm{CH}_{R}\right)$; their crossing formed the lateral border. For the medial border, the right cheilion was connected with the right inner canthus following the nasolabial fold. All connecting curves followed the surface. The same procedure was repeated for the left side.

B: Histogram of log-transformed normalized signature for the malar region of patients compared to controls.

C: Signature graph for differences along the anterior-posterior axis for the malar region. Each malar signature is annotated by an outline of a different colour depending on its pattern label: BP-green; EF-black; LLA-blue; SC-red.

D: Collapsed version of signature graph shown in C using the same colour coding of pattern labels.

\title{
Figure 3 Facial asymmetry signatures and associated graph for patients
}

\author{
A. Heat mapped face asymmetry signatures of 41 patients of Dutch descent. Each \\ demarcates differences of original and reflected surfaces for each patient normalized to \\ similar differences of matched controls using a red/green/blue scale for "below -2SD" /zero \\ $\mathrm{SD} /$ "over +2SD". Each heat map will necessarily be anti-symmetric in its red-blue colouring \\ since the right sided differences of original surface face compared to its reflection or \\ mirrored form will be the opposite for the left side.
}


B. Signature graph for the signatures in A. Colour outlines are used to identify the pattern subgroup to which an individual belongs: BP-black; EF-red; LLA-green; SC-blue.

John Wiley \& Sons, Inc. 
3D Morphometry Aids Facial Analysis of Individuals with a Childhood Cancer

Saskia M. J. Hopman ${ }^{1}$, Johannes H. M. Merks ${ }^{1}$, Michael Suttie ${ }^{2}$, Raoul C. M. Hennekam ${ }^{3}$, Peter Hammond ${ }^{2}$

1: $\quad$ Department of Pediatric Oncology, Emma Children's Hospital, Academic Medical Center, Amsterdam, Netherlands

2: $\quad$ Genetics \& Genomic Medicine, UCL Institute of Child Health, London, UK

3: Departments of Pediatrics and Clinical Genetics, Emma Children's Hospital, Academic Medical Center, Amsterdam, Netherlands

Running title: 3D Analysis in Childhood Cancer Patients

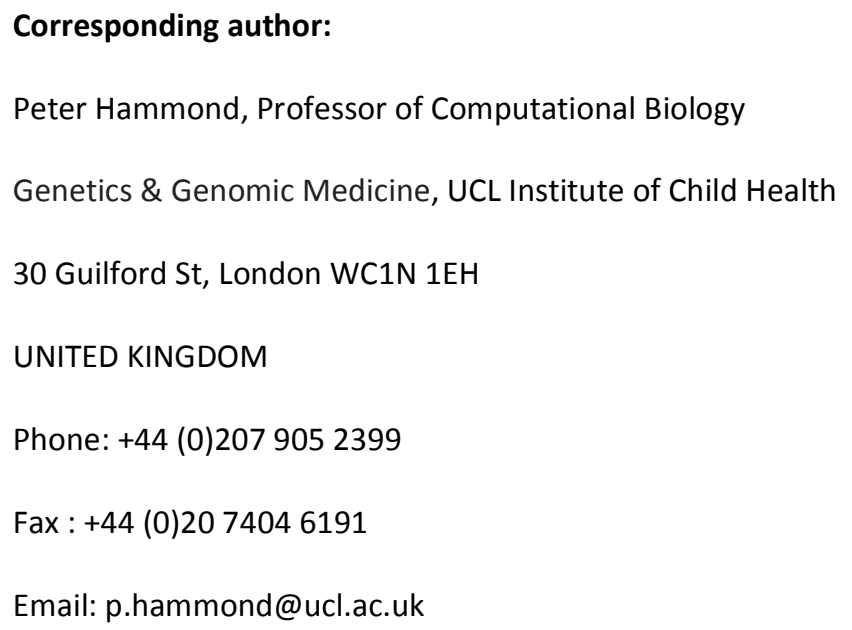




\begin{abstract}
A group of patients who had cancer as a child were previously found to have distinct patterns of morphological abnormalities. In this study, we investigated the added value of 3D shape analysis to characterize their facial morphology.

Primarily, we showed in an objective and quantitative manner that the overall facial dysmorphism of the individuals who had had a childhood cancer was significantly greater than that of the controls. We also demonstrated how the same approach can be used to detect a similar disparity for a more localized malar region comprising customized disconnected patches defined on both sides of the face. In addition, by comparing original face surfaces to their mirrored forms, we confirmed that the patient group had significantly greater facial asymmetry than the controls. Each of these results made use of surface shape differences not detectable by simple linear or angular characteristics as might be used in analyses based on measures captured manually or derived from landmarks annotating 2D photographic images.

We conclude that 3D morphometric analysis of a relatively small heterogeneous patient group can further delineate face shape differences from typically developing individuals that are too subtle or geometrically complex to identify or quantify objectively with conventional clinical and anthropometric approaches.
\end{abstract}


Hopman et al. 3D Analysis in Childhood Cancer Patients

\section{KEYWORDS}

Three-dimensional imaging; 3D; morphometric analysis; morphological abnormalities; face shape differences; childhood cancer 


\section{INTRODUCTION}

Many genetic syndromes involve facial morphological characteristics, and the facial 'Gestalt' can be an important clue in the identification of genetic conditions [Hammond, 2007; Hammond et al., 2012b; Hennekam et al., 2010]. We also know that in tumor predisposition syndromes, the constitutional molecular defects that lead to oncogenesis may also play an important role in facial development [Ponder, 2001]. Traditionally, anthropometry has been an important tool in analyzing the phenotype of an individual [Farkas, 1994]. It has the disadvantage of inter-observer variability and requires prolonged co-operation of the subject as well as their presence for capturing additional measurements. This limits the use of anthropometry on children or individuals with an intellectual disability. Two-dimensional photography overcomes some of these limitations, but the quality and usability of a 2D picture is highly dependent on the experience of the photographer and cooperation, pose and lighting of the subject [Hammond, 2007].

Three-dimensional (3D) photogrammetric images are independent of pose, and offer repeat measurement without the subject's presence or prolonged cooperation [Hammond et al., 2012a]. Typically, 3D face images are annotated with anatomical landmarks to produce linear and angular measurements. For facial analysis, dense surface modelling (DSM) techniques induce tens of thousands of densely corresponded points as quasilandmarks from a small number of manually placed landmarks. Using DSMs, the average surface of a group of faces can be computed and quantitative shape comparisons of face surfaces can be performed between individuals or relative to ethnically, age- and sexmatched means [Hammond \& Suttie, 2012a]. DSM based analysis has successfully delineated the facial phenotype of a variety of neurodevelopmental conditions [Hammond 
et al., 2005; Hammond et al., 2008].

In a previous study, we used 'traditional' anthropometry and morphological examination in a cohort of 1,073 individuals who had cancer as a child, and compared these to 1,007 healthy schoolchildren [Merks et al., 2005]. We demonstrated significantly increased incidence of morphological abnormalities and high prevalence of syndromes. Furthermore, we described four new patterns of co-occurring morphological abnormalities as tumor predisposition syndromes [Merks et al., 2008] within this cohort. These were named after key abnormalities: "blepharophimosis (BP) pattern", "epicanthal folds (EF) pattern", "asymmetric lower limbs (LLA) pattern" and "Sydney creases (SC) pattern" (Table I). The structural genomic variants identified in patients showing one of the four patterns were reported recently [Hopman et al., 2013a]. The goal of the present study is to evaluate whether using 3D shape analysis in this same subgroup of childhood cancer patients adds to the evaluation of facial morphology by clinical inspection. More specifically, to determine objectively if there is a difference in dysmorphisms observed between childhood cancer patients and healthy controls. Additional objectives are to objectively determine the shape of a geometrically complex facial region, i.e. the malar region, that previously could be evaluated only subjectively, and to identify additional morphological signs within the patient group that were previously undetected by clinical inspection. We hypothesize that childhood cancer patients show more dysmorphisms compared to healthy controls (regardless effects of therapy modalities). 


\section{Image capture and preparation}

3D facial imaging was undertaken using commercial stereo-photogrammetric cameras able to capture more than 25,000 3D points on an adult face surface (Canfield Imaging Systems, New Jersey, USA; 3dMD, Atlanta, USA). Each face image was manually annotated using in-

\begin{tabular}{l} 
Formatted: Font: +Body (Calibri), $12 \mathrm{pt}$ \\
\hline Formatted: Font: +Body (Calibri), $12 \mathrm{pt}$ \\
\hline Formatted: Font: +Body (Calibri), $12 \mathrm{pt}$ \\
\hline Formatted: Font: +Body (Calibri) \\
\hline
\end{tabular}

current study,-we confined our analyses to subjects of self-reported Dutch descent[Hopman et al., 2013b]. Forty-nine childhood cancer patients, previously designated as showing one of four patterns of morphological abnormalities [Merks et al., 2008; Hopman et al., 2013a] were asked to participate and only one declined. In this cohort, there were no patients who had undergone local therapy in the facial region and all patients had finished their cancer therapy for more than five years. The 3D photos of $7 / 48$ patients were excluded for technical reasons or due to their non-Dutch descent [Hopman et al., 2013b]. The characteristics of the patient subgroups are shown in Table II.

The control group consisted of 274 subjects of self-reported Dutch descent recruited from university scientific and hospital medical professionals $(81 / 274,30 \%)$ and unaffected parents and sibs of children with a molecularly proven genetic syndrome who visited outpatient departments or attended family support group meetings (193/274, 70\%). The composition and average ages of patient and control groups are shown in Table III. 
house software with 22 landmarks previously shown to be reliable and reproducible [Gwilliam et al., 2006]. The landmarks were used to induce a dense correspondence across all subjects of all $25,000+$ surface points. A DSM of all faces in the dataset was generated as the set of principal component analysis (PCA) modes covering $99 \%$ of shape variation of face shape difference from the overall mean face. DSM construction involved methods developed in-house which are described elsewhere [Hutton, 2004; Hammond et al., 2005; Hammond \& Suttie, 2012a].

Subjective morphological signs that show differences in a specific direction were evaluated along an appropriate axis (lateral, vertical, anterior-posterior) and in a model confined to a region containing the sign under study [Hammond \& Suttie, 2012a]. Separate DSMs of the reduced face without ears and the malar region (Figure 2A) were computed. Ears were omitted because of their frequent partial, or occasional complete, occlusion by hair.

\section{Statistical analyses}

For the continuous outcome variables with a normal distribution, student-t-tests were performed using a significance level of 0.05 . For t-tests, only the controls in the patient defined age range of 10 to 40 years were included. Anthropometric calculations and t-tests were performed in Excel (Microsoft Office 2010) and StatPlus (AnalystSoft, version 5.7.5). In order to maximize the analysis of the relatively small number of female patients, male and female asymmetry measures were adjusted for sex and age based on the control population before combined analysis. Age adjustment was achieved using separate age-based linear regressions for male and female controls, and the formula:

$$
\text { adjusted }=(\text { orginal }-m * \text { age-c }) / \text { sqrt }(1+m * m)
$$


where $y=m^{*} x+c$ is the equation of the regression line for original against age. Following age adjustment, z-scores were computed based on means and standard deviations for the appropriate age range and same sex controls.

Bootstrap analyses were performed to sample estimates of the difference in the mean asymmetry indices, mean overall facial dysmorphism scores and mean dysmorphism score for the malar region respectively. We compared the means of for each of these values for patients and controls with 1000 bootstrap samples. The overall facial dysmorphism scores dysmorphism scores for the malar region and asymmetry indices were adjusted for sex, age and ethnicity. As a measurement of effect size, we calculated the Cohen's D value as the difference between the means divided by the pooled SD for overall facial dysmorphism scores dysmorphism scores for the malar region and asymmetry indices.

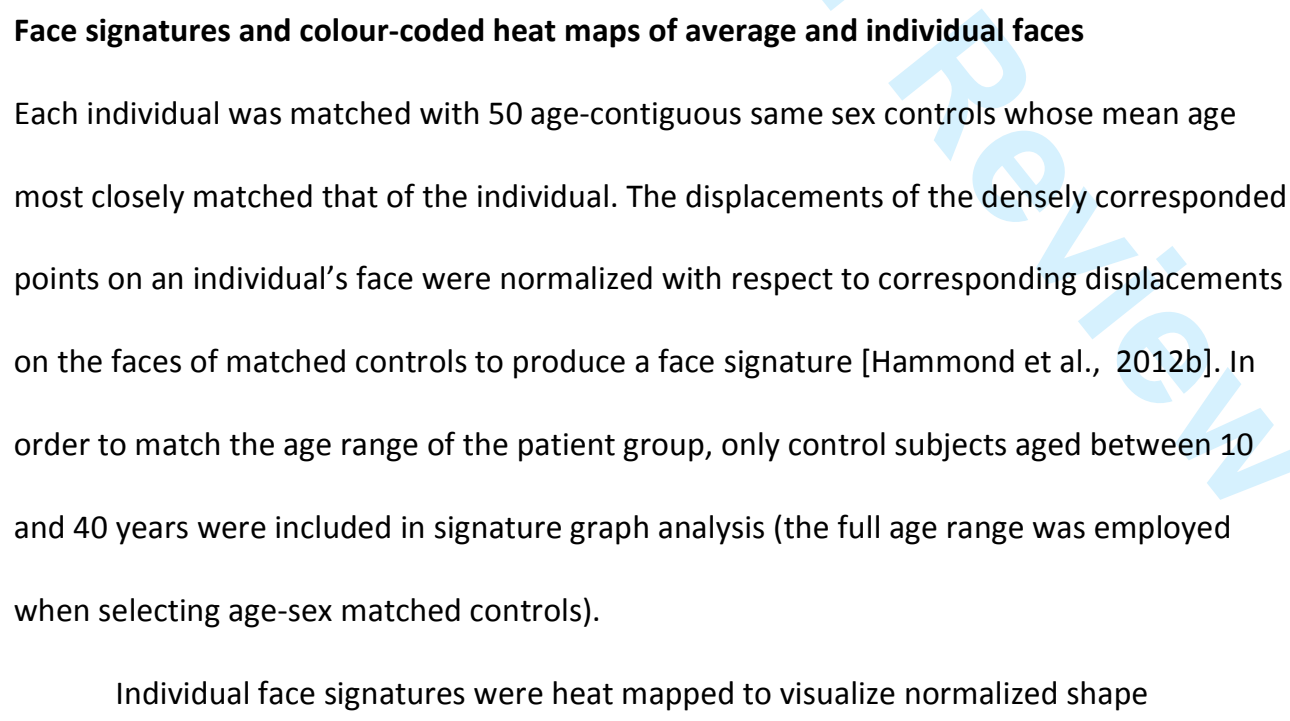


corresponded to contraction/coincidence/expansion of the surface being compared.

Analogous processes produce signatures for surface shape differences parallel to lateral, vertical, and depth (anterior-posterior) axes. The signature weight of an individual face or face patch (square root of the sum of the squared normalized differences for all densely corresponded points) reflects the deviation of a subject from matched controls and thus defines a useful estimate of the facial dysmorphism of an individual.

A face signature graph is constructed using face signatures as vertices or nodes and a directed edge links each vertex to its most "similar" signature. An alternative, but topologically identical, "coloured" form of a face signature paints the vertices to reflect a diagnostic or other subgroup labelling such as control-patient dichotomy or tumor disposition pattern. Finally, a "coloured" signature graph can be "collapsed" to a simplified form where vertices represent clusters of same-coloured connected vertices of the original [Hammond \& Suttie, 2012a]. An entropy-like measure is used to summarize the associated clustering or dispersion of the members of each labelled subgroup. The lower the dispersion factor of a labelled subgroup, the stronger is the similarity of the facial dysmorphism of subcluster members compared to other subgroups. A maximum dispersion factor of 1.00 means that a subgroup's members are perfectly dispersed into singleton subsets and so do not cluster at all because they lack homogeneity and differ from other labelled subgroups in their dysmorphism. All signature graphs were drawn using the open source software GraphViz (version 2.26, www.graphviz.org).

\section{Facial asymmetry}

As in previous studies of facial asymmetry, we constructed a DSM containing the original and reflected (mirrored form) of the face surfaces of all study participants. The manually 
placed and left-right-paired landmarks were swapped over before resampling the surface, effectively interpolating a dense set of landmarks on both sides of the face. Thus, the interpolated landmarks are matched-paired across the face; to study face asymmetry, a DSM was constructed from the combined set of densely corresponded face surfaces and their mirrored forms. We estimated individual facial asymmetry as the Euclidean distance between the DSM representations of the original and mirrored face surfaces. [Hammond et al., 2008].

This was adjusted to take age and sex into consideration since there are gender differences and asymmetry tends to increase with age within the general population [Hammond et al., 2008; Claes et al., 2012a].

\section{RESULTS}

1. The individuals with a childhood cancer have greater facial dysmorphism than age-sexethnicity matched controls but no set of common dysmorphic features, not even within the tumor predisposition patterns found previously

The heat mapped signatures in Figure $1 \mathrm{~A}$ delineate localized individual facial dysmorphism of 12 female (row 1) and 29 male patients of Dutch descent (rows 2 to 4). This visualization of detailed face shape differences offers an objective adjunct to clinical examination. It is possible to identify small subgroups of patients with a similar heat map highlighting similar facial dysmorphism such as malar flattening (anterior red patches on cheeks of LLA_9, LLA_24, LLA_34, LLA_43), perioral insufficiency (red regions around mouths of LLA_29, LLA_45, SC_3, SC_26, LLA_29) and mid-facial hyperplasia (blue patches on midface of EF_2, EF_7, LLA_20). However, overall, there is considerable heterogeneity in the facial dysmorphism of the patient group. This is confirmed in the collapsed form of the face 
signature graph for controls and patients (Figure 1C) with dispersion factors of 0.94 for patients and 0.16 for controls, and the patient group's clustering as a large number of singletons, a few duos and a quartet.

The location of patients in the periphery or as terminal nodes of the signature graph for controls and patients is as a result of their greater facial dysmorphism (Figure 1B). Furthermore, the associated (log transformed) face signature weight distribution (Figure 1D) confirms a significantly greater mean for patients $(M=2.20)$ compared to controls $(M=2.14)$; $t(54)=2.35, p=0.02$.

The bootstrap analysis shows a difference of $0.67(95 \% \mathrm{Cl} 0.014-0.124, \mathrm{p}<0.05)$ between the mean overall facial dysmorphism score for patients and controls. The Cohen's d value for effect size of the overall facial dysmorphism score (adjusted for sex, age and ethnicity) between patients and controls is 0.4 (i.e. between medium and large effect size). Inspection of the face signatures in Figure $1 A$ suggests the LLA pattern subgroup to have more red-blue hues than green and hence to be more facially dysmorphic. Some quantitative evidence for this is seen in a control vs pattern histogram of signature weights (Supplementary Figure 1) but the relatively low numbers in the other pattern subgroups rather undermines such cross pattern comparisons.

\section{The malar region is more dysmorphic in patients compared to controls}

Although, as shown above, there was no discernible set of common dysmorphic features in the patient group when considering the face in its entirety, the malar region is notably painted red or blue (at 2SD) in a majority of the heat mapped signatures of Figure $1 A$. This clinical observation suggested that the malar region should undergo closer objective 
scrutiny. Given the complex geometry of the malar region, this would be impossible using linear or angular measures derived solely from landmarks. In order to investigate malar dysmorphism, a customized region was demarcated as depicted in Figure $2 \mathrm{~A}$.

The distribution of signature weight as a measure of dysmorphism for the malar region is shown in the histogram in Figure 2B. The malar dysmorphism score is indeed significantly higher in patients $(M=1.81)$ compared to controls $(M=1.74) ; t(58)=2.93, p=0.004$. The bootstrap analysis shows a difference of $0.52(95 \% \mathrm{Cl} 0.019-0.127, \mathrm{p}<0.05)$ between the mean dysmorphism score of the malar region for patients and controls. The Cohen's d value for effect size of the dysmorphism score for the malar region (adjusted for sex, age and ethnicity) between patients and controls is 0.5 (i.e. between medium and large effect size).

The collapsed signature graph for the malar region for controls and patients, as was the case for the face, shows patients to be located more at the periphery, reflecting greater malar dysmorphism. The malar region dispersion factors for controls (0.16) and patients (0.92) also reflect the heterogeneity of the malar dysmorphism for the patients when considered as a whole (Supplementary Figure 2).

Interestingly, the signature graph for differences along the anterior-posterior or zaxis of the malar region for patients alone shows some stronger clustering for LLA patients compared to other pattern subgroups (Figure $2 C$ ). This is more clearly shown in the collapsed form of the signature graph and the lower dispersion factor (0.66) for LLA-pattern patients (Figure 2D). In comparison, EF-, SC- and BP-pattern patients show dispersion factors of $0.83,0.87$ and 1.00 respectively. This suggests that the LLA-pattern subgroup of these 41 patients are more similar in their malar flattening or malar prominence than patients from the other pattern subgroups (Figure 2D). 


\section{Patients have significantly greater facial asymmetry when compared to controls}

The PCA-vector representation of each original face surface was subtracted from that for its mirrored form in the DSM generated for all original and mirrored or reflected images. The "size" of this vector difference is an estimate, or index, of the overall asymmetry of the original face surface. Facial asymmetry increases with age and at different rates for males and females as is shown by the separate male and female scatter plots for the raw asymmetry index against age (Supplementary Figures $3 A$ and 3B)[Hammond et al., 2008]. When adjusted for age (linear regression adjusted) and z-scored for gender within the approximate patient age range of 10 to 40 years, there was significantly greater asymmetry in patients $(M=0.75)$ compared to controls $(M=0 ; t(52)=2.2, p=0.03)$. The bootstrap analysis shows a difference of $0.8006(95 \% \mathrm{Cl} 0.17-1.57, \mathrm{p}<0.05)$ between the mean adjusted asymmetry indices for patients and controls. The Cohen's d value for effect size of the asymmetry index (adjusted for sex, age and ethnicity) between patients and controls is 0.4 (i.e. between medium and large effect size).

This greater facial asymmetry for patients compared to controls can also be appreciated on an individual basis by interpreting the PCA vector difference between original and mirrored DSM PCA vectors as a surface in the DSM model. The resulting original-reflected difference for each patient can then be normalized against corresponding control differences just as for signatures for the original faces. Thus, corresponding to the gallery of face signatures of Figure 1 is another showing normalized asymmetry differences from the asymmetry of age-sex matched controls in the form of heat mapped facial asymmetry signatures (Figure $3 A$ ). Once again, it is possible to discern subgroups, this time with similar asymmetry dysmorphism. This is more easily observed by considering the 
induced clustering in the asymmetry signature graph of the patients alone (Figure $3 B$ ). Of the seven subclusters within the graph, subcluster 2 contains a duo of faces showing almost no difference (mostly green hue) from the asymmetry of age-sex-ethnicity matched controls. Subcluster 3 contains 8 individuals displaying a right dominant asymmetry difference from controls in the supraorbital region (blue above right eye, red above left eye). Indeed, the faces in subclusters 3, 4 and 5, below and to the left of subcluster 2, largely show right dominant asymmetry differences from controls, whereas subclusters 1, 6 and 7 , above and to the right of subcluster 2, largely include individuals with more left dominant asymmetry. It also noticeable that most mandibular asymmetry occurs in the EF pattern. However, the small sizes of the pattern subgroups in this patient population somewhat undermines such attempts to hypothesize about intra-pattern similarities and inter-pattern differences.

\section{DISCUSSION}

The main purpose of this study was to identify additional benefits that 3D facial morphometry might offer beyond the clinical and classical anthropometric facial analysis carried out in the origin Merks et al study [Merks et al., 2005] of more than 1,000 individuals who had suffered a childhood cancer and a similarly sized group of controls. In the previous study, tumor predisposing syndrome patterns were identified by considering not just the face but other physical attributes as well. Inevitably, in this study of only faces, and in less than $3 \%$ of the original patient cohort, it was unlikely that the original patterns could be confirmed or that novel patterns might emerge. With the future study in mind of tens of thousands of individuals who have had a childhood cancer, it was more important that this study determine how best to exploit 3D facial morphometric analysis in the future 
on a much larger scale and in a much more heterogeneous population than has generally been undertaken hitherto.

Rather than investigate individual linear or angular measures derived from landmarks, we employed signature based analysis where surface shape differences at 25,000+ surface points are normalized against age-sex-ethnicity matched controls for the whole face, for face patches and for the difference between an original face and its mirrored or reflected form. We used heat maps of the signatures to delineate individual localized dysmorphism. The "weight" of the signatures was used to estimate an index of dysmorphism, through which we established statistically significant differences between the patients and controls within the same age range. Signature graphs with directed edges linking most similar signatures were used to cluster both controls and patients into subsets with similar dysmorphism i.e. shape differences from age-sex matched controls. Relative central or peripheral position in signature graphs reflected low or high levels of dysmorphism and, in the collapsed form of signature graphs, rates of dispersion quantified overall clustering rates of patients compared to controls and/or between pattern subgroups of patients.

Using the methodological approach summarized above, we detected significantly higher dysmorphism scores (signature weights) for the facial region without ears in the individuals who had cancer as a child. This was also reflected in the corresponding signature graph for controls and patients where the latter patients appeared more in the periphery and as terminal nodes. Common facial differences between patients and controls could not be identified and a high dispersal rate, reflecting lack of similarity clustering of the patient group, provided supporting evidence. Similarly, when considering patients on their own, no intra-pattern similarities or inter-pattern differences were evident in the signatures or the signature graphs._Based on subjective evaluation of the face signatures, we hypothesized 
that the malar region of patients was more dysmorphic than in controls. This would be a challenging hypothesis to test using linear or angular measures derived from clinical examination or derived from landmarked photographs. It required an analysis of 3D surface shape. An experienced dysmorphologist (RCMH) defined a customized region to act as the focus for analyzing shape differences in the malar region and as a base shape for constructing DSMs. Following the same methodological sequence as for face shape analysis, we established significantly greater dysmorphism of the malar region in the patients compared to controls. Once again, shape signature graphs were used to confirm this. In terms of intra-pattern analysis of anterior-posterior malar shape difference, the LLA pattern subgroup produced a lower dispersion rate suggesting some commonality of form for malar flattening or prominence. We should realize that typically in evolutionary, developmental and systematic biology, we use geometric morphometrics to measure form and its variations. These approaches are often unsuitable for describing phenotypes expressing discordant shape variation that do not occur in the normal population at all, not even under extreme forms. Ultimately, it requires extension to geometric morphometrics to quantify abnormal shape variation and with that, a redefinition of what is called dysmorphism. Claes et al. demonstrate the use of so called dysmorphometrics to measure unusual form $\underline{\text { differences given only normative data. It builds upon existing geometric morphometric }}$

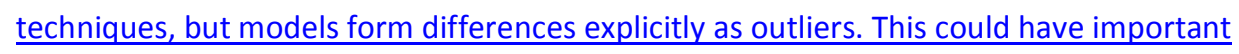
implications for biomedical practice and research [Claes et al., 2012b]. could have been an effect of therapy that childhood cancer patients may have received. However, patients who had undergone radiotherapy in the facial region were excluded. Therefore, the increased facial asymmetry in patients is more likely to reflect 'intrinsic 
asymmetry' rather than asymmetry caused by external effects. We should note that the

Formatted: Font: $12 \mathrm{pt}$, Not Italic asymmetry analyses we performed in this paper do not include a test for significance of asymmetry over measurement error. Previous studies have considered fluctuating asymmetry: nondirectional deviations from bilateral symmetry [Palmer and Strobeck 1986], including a procedure to make bilateral variation serve as a measurement of developmental precision [Palmer and Strobeck 1997]. This was explored further in terms of the heritability of fluctuating asymmetry [Klingenberg et al., 1999] and on shape analysis of symmetric structures to quantify asymmetry (and variation) among individuals [Klingenberg et al., 2002]. It is recommended that in asymmetry analyses, tests for significance of asymmetry (above measurement error), directional asymmetry (side-bias) and deviation from normality (antisymmetry) are incorporated. They propose a framework to do so [Klingenberg et al., 2002; Klingenberg 2015] for asymmetry analyses of paired simple measures or for tens of left-right paired landmarks. The 3D asymmetry estimate in our study is based on PCA mode scores for 3D surfaces comprising 25,000+ unpaired surface points. At this point, it is unclear to us how the proposed methodology might be applied to our situation. This is an important omission to be addressed in the literature.

Asymmetry occurs frequently in genetic conditions associated with a malignancy. For example, it is a well-known feature in Bannayan-Riley-Ruvalcaba syndrome (BRRS, OMIM: 153480) [Bannayan, 1971] and Beckwith-Wiedemann Syndrome (BWS, OMIM: 13060) [Wiedemannnn, 1964]. It should be noted that none of the patient subgroup of this study were diagnosed with established syndromes such as BRRS or BWS. Some potential for interand intra-pattern analysis of facial asymmetry was identified for particular parts of the face, e.g. supra-orbit and mandible, and may assist in future determination of face shape differences and even alternative tumor disposition patterns of dysmorphism. 
In this project, we used 3D facial photographs and dense surface modelling morphometric techniques to characterize the facial phenotype of a cohort of patients who had cancer as a child, and in whom distinct patterns of morphological abnormalities were previously demonstrated using physical examination and anthropometry [Merks et al., 2008]. The patterns consist of abnormalities located both in the face and in other body parts. As we used only the face, it is impossible to evaluate the complete morphology of patterns this way. Therefore, it was impossible to repeat the grouping of the patients using signature graphs for the complete cohort (1,073 childhood cancer patients) described by Merks et al. [Merks et al., 2008], blinded from the statistical patterns of co-occurring morphological abnormalities. The relatively small number of individuals of the various patterns and in the total cohort hampers detailed statistical analyses and prevents some subgroup analysis. Another overall limitation of our analysis could be that, in computing face signatures, the age- and sex-matched controls selected are according to a matching running mean age, which could be biased by lack of coverage of certain ages.

Despite these limitations, the 3D face image capture and subsequent analysis has shown significant added value. We conclude that dense surface modelling techniques expand the possibilities for physicians (plastic surgeons, clinical geneticists etc.) to describe and characterize the phenotype of an individual or group of individuals and allow objective comparisons to age- and sex-matched controls. The addition of the approach adopted here in a large, nation-wide study of individuals who have had cancer as a child, together with a recently developed tool to recognize individuals with a high chance to have a tumor predisposition syndrome [Hopman et al., 2013c] will allow recognition of larger subgroups within pediatric cancer patients. Signature graphs can help pre-sorting the patients into clusters with similar differences from the control group and allow further characterization of 
the faces and facilitate subsequent molecular analyses. Such a study is presently in preparation.

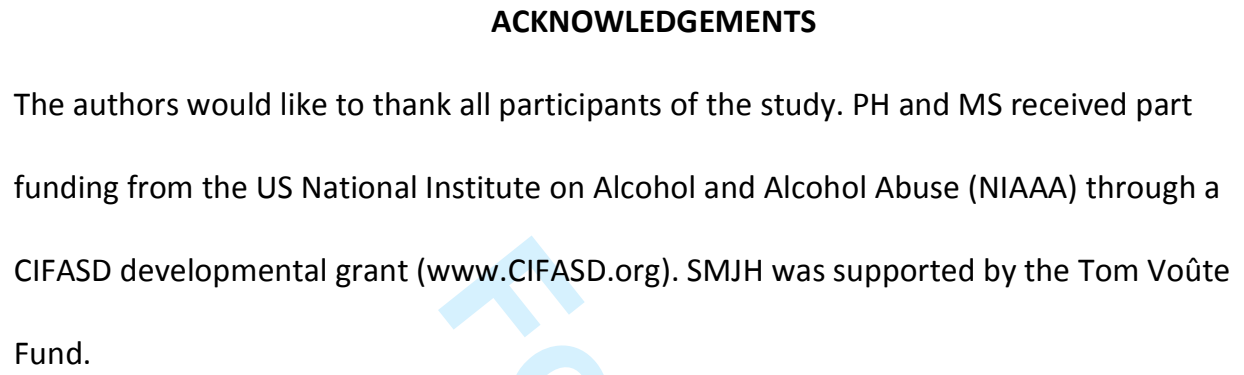




\section{SEPARATE BOX}

\section{DEFINITIONS AND EXPLANATION OF FREQUENT USED TERMS ${ }^{\mathrm{a}}$}

LANDMARKS: Designated soft tissue points on anatomically well-defined locations.

EUCLIDEAN DISTANCE: Mathematic term for distance between two $\mathrm{n}$-dimensional vectors defined as square root of sum of $\mathrm{n}$ squared component-wise differences.

DENSE SURFACE MODEL (DSM): The actual 3D model, which forms the basis for further 3D analysis. A DSM is built from a set of surfaces of which mean landmarks are calculated using the Procrustes algorithm. The surfaces of the individual subjects are warped to the mean landmarks, aligning them. The points on a selected face can be mapped to the closest points on each face to induce a dense correspondence of tens of thousands of images across all images. The DSM is formed from the principal components covering $99 \%$ of shape variation from the overall average based on the densely corresponded points.

FACE SIGNATURE: The normalized differences, typically orthogonal to the face surface, of surface point displacements from corresponding positions of the densely corresponded points on the mean surface.

SIGNATURE WEIGHT: The square root of the sum of the squared normalized differences for all densely corresponded points.

DYSMORPHISM SCORE: The deviation of a subject from matched controls calculated by the signature weight of an individual face or face patch (square root of the sum of the squared normalized differences for all densely corresponded points). 
FACE SIGNATURE DISTANCE (FSD): An automated partial ordering of face signatures is defined as the Euclidean distance between the vectors representing the normalized differences across the densely corresponded points. A face signature graph can then be constructed with a set of face signatures as its vertices. A directed edge is drawn from each signature to another, possibly not unique, signature with the smallest FSD from the first,or equivalently the most similar dysmorphism to the first. The length of an edge between two vertices is the FSD between them. The shorter the edge, the more similar is the nature of the facial dysmorphism of the linked faces.

SIGNATURE GRAPH: Graphical representation of a set of face signatures as vertices. A directed edge is drawn from one signature to another signature with the smallest FSD from the first. In a signature graph, the FSD is the length of the edge between two vertices.

LINEAR MEASUREMENTS: Calculations of anthropometric distances using the 3D coordinated of anatomical landmarks.

adapted from [Hammond \& Suttie, 2012a] and [Hammond et al., 2012b] 


\section{REFERENCES}

Allanson JE, Biesecker LG, Carey JC, Hennekam RC. 2009a. Elements of morphology: introduction. Am.J.Med.Genet.A 149A: 2-5.

Allanson JE, Cunniff C, Hoyme HE, McGaughran J, Muenke M, Neri G. 2009b. Elements of morphology: standard terminology for the head and face. Am.J.Med.Genet.A 149A: 6-28.

Bannayan GA. 1971. Lipomatosis, angiomatosis, and macrencephalia. A previously undescribed congenital syndrome. Arch.Pathol. 92: 1-5.

Biesecker LG, Aase JM, Clericuzio C, Gurrieri F, Temple IK, Toriello H. 2009. Elements of morphology: standard terminology for the hands and feet. Am.J.Med.Genet.A 149A: 93-127.

Carey JC, Cohen MM, Jr., Curry CJ, Devriendt K, Holmes LB, Verloes A. 2009. Elements of morphology: standard terminology for the lips, mouth, and oral region. Am.J.Med.Genet.A 149A: 77-92.

Claes P, Walters M, Shriver MD, Puts D, Gibson G, Clement J, Baynam G, Verbeke G, Vandermeulen D, Suetens P. 2012a. Sexual dimorphism in multiple aspects of 3D facial symmetry and asymmetry defined by spatially dense geometric morphometrics. J.Anat. 221:

Formatted: Dutch (Netherlands) 97-114.

Claes P, Daniels K, Walters M, Clement J, Vandermeulen D, Suetens P. 2012 b.

Formatted: Dutch (Netherlands)

Dysmorphometrics: the modelling of morphological abnormalities. Theoretical Biology and

Formatted: English (U.S.)

Medical Modelling, 9: 5

Formatted: English (U.S.)

Formatted: English (U.S.)

Farkas L. 1994. New York: Raven Press. Anthropometry of the head and face in medicine. 
Gwilliam JR, Cunningham SJ, Hutton T. 2006. Reproducibility of soft tissue landmarks on three-dimensional facial scans. Eur.J.Orthod. 28: 408-415.

Hall BD, Graham JM, Jr., Cassidy SB, Opitz JM. 2009. Elements of morphology: standard terminology for the periorbital region. Am.J.Med.Genet.A 149A: 29-39.

Hammond P. 2007. The use of 3D face shape modelling in dysmorphology. Arch.Dis.Child 92: $1120-1126$

Hammond P, Forster-Gibson C, Chudley AE, Allanson JE, Hutton TJ, Farrell SA, McKenzie J, Holden JJ, Lewis ME. 2008. Face-brain asymmetry in autism spectrum disorders.

Mol.Psychiatry 13: 614-623.

Hammond P, Hutton TJ, Allanson JE, Buxton B, Campbell LE, Clayton-Smith J, Donnai D, Karmiloff-Smith A, Metcalfe K, Murphy KC, Patton M, Pober B, Prescott K, Scambler P, Shaw A, Smith AC, Stevens AF, Temple IK, Hennekam R, Tassabehji M. 2005. Discriminating power of localized three-dimensional facial morphology. Am.J.Hum.Genet. 77: 999-1010.

Hammond P, Suttie M. 2012a. Large-scale objective phenotyping of 3D facial morphology. Hum.Mutat. 33: 817-825.

Hammond P, Suttie M, Hennekam RC, Allanson J, Shore EM, Kaplan FS. 2012b. The face signature of fibrodysplasia ossificans progressiva. Am.J.Med.Genet.A 158A: 1368-1380.

Hennekam RC, Allanson JE, Biesecker LG, Carey JC, Opitz JM, Vilain E. 2013. Elements of morphology: Standard terminology for the external genitalia. Am.J.Med.Genet.A 161: 12381263. 
Hennekam RC, Cormier-Daire V, Hall JG, Mehes K, Patton M, Stevenson RE. 2009. Elements of morphology: standard terminology for the nose and philtrum. Am.J.Med.Genet.A 149A: 61-76.

Hennekam RCM, Krantz ID, Allanson JE. 2010. Gorlin's syndromes of the head and neck. (5th ed.) New York, USA: Oxford University Press Inc.

Hopman S, Merks J, Eussen H, Douben H, Snijder S, Hennekam R, de KA, Caron H. 2013a. Structural genome variations in individuals with childhood cancer and tumour predisposition syndromes. Eur.J.Cancer. 4: 2170-8.

Hopman S, Merks J, Suttie M, Hennekam R, Hammond P. (2013b). Face shape differs in Formatted: Space After: $12 \mathrm{pt}$ phylogenetically related populations. Eur J Hum Genet. 22: 1268-71.

Hopman SM, Merks JH, de Borgie CA, Aalfs CM, Biesecker LG, Cole T, Eng C, Legius E, Maher ER, van Noesel MM, Verloes A, Viskochil DH, Wagner A, Weksberg R, Caron HN, Hennekam RC. 2013c. The development of a clinical screening instrument for tumour predisposition syndromes in childhood cancer patients. Eur.J.Cancer. 49: 3247-54.

Hunter A, Frias JL, Gillessen-Kaesbach G, Hughes H, Jones KL, Wilson L. 2009. Elements of morphology: standard terminology for the ear. Am.J.Med.Genet.A 149A: 40-60.

Hutton TJ. (2004). Dense surface models of the human face. Thesis/dissertation University

Formatted: Space After: $12 \mathrm{pt}$ College London, London.

\section{Klingenberg CP, Nijhout HF. 1999. Genetics of fluctuating asymmetry: a developmental} model of developmental instability. Evolution. 53: 358-375. 
Klingenberg CP, Barluenga M, Meyer A. 2002.Shape analysis of symmetric structures:

quantifying variation among individuals and asymmetry. Evolution.56:1909-1920.

Klingenberg CP. 2015. Analyzing fluctuating asymmetry with geometric morphometrics: concepts, methods and applications. Symmetry. 7: 843-934.

Merks JH, Caron HN, Hennekam RC. 2005. High incidence of malformation syndromes in a series of 1,073 children with cancer. Am.J.Med.Genet.A 134A: 132-143.

Merks JH, Ozgen HM, Koster J, Zwinderman AH, Caron HN, Hennekam RC. 2008. Prevalence and patterns of morphological abnormalities in patients with childhood cancer. JAMA 299: 61-69.

Palmer AR, Strobeck C. 1986. Fluctuating asymmetry: measurement, analysis and patterns. ann. Rev. Ecol. Syst. 17: 391-421.

Palmer AR, Strobeck C. 1997. Fluctuating asymmetry and developmental stability: heritability of observable variation vs. heritability of inferred cause. J. Evol. Biol. 10: 39-49.

Ponder BA. 2001. Cancer genetics. Nature 411: 336-341.

Wiedemann HR. 1964. Familial malformation complex with umbilical hernia and 


\section{TABLES}

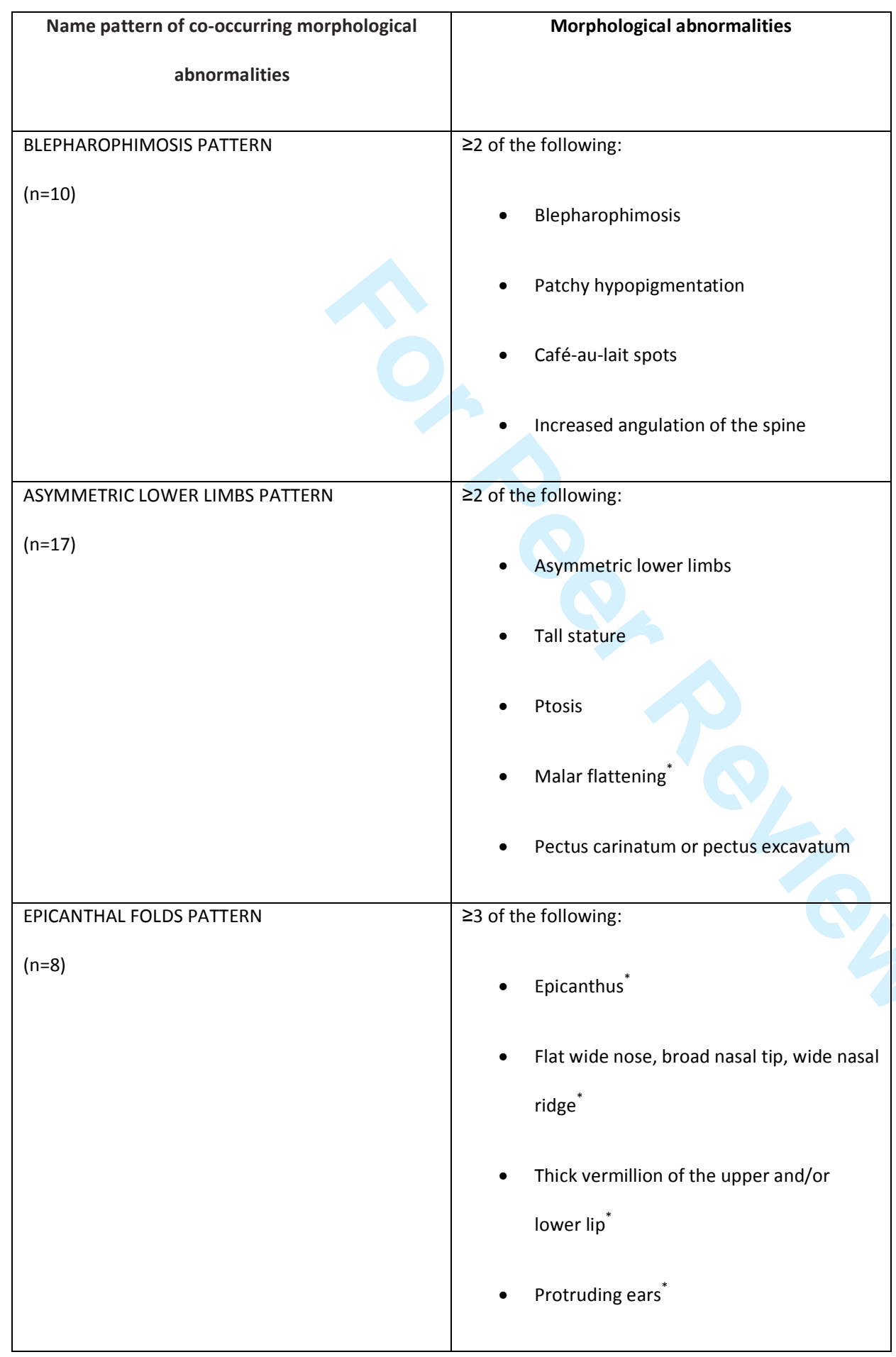




\section{Table I: Summary of patterns of statistically significant co-occurring morphological}

abnormalities and number of patients in each pattern for 3D analyses. In the current study 41 patients were available for 3D facial analyses. The patients from the current cohort were selected from a large prospective cohort of childhood cancer patients and all showed one of the four patterns of statistically significant co-occurring morphological abnormalities. The table shows the criteria for each of the patterns and the number of patients included from each pattern, based on the morphological examination by Merks et al. (partly described in [Merks et al., 2008]). This table is adapted from [Hopman et al., 2013a].

*= Modified according to Elements of Morphology nomenclature [Allanson et al., 2009a; Hunter et al., 2009; Hennekam et al., 2013; Biesecker et al., 2009; Allanson et al., 2009b; Carey et al., 2009; Hennekam et al., 2009; Hall et al., 2009]. 


\begin{tabular}{|c|c|c|c|}
\hline SEX & PATTERN & SUBJECTS & MEAN AGE \\
\hline \multirow{4}{*}{$\begin{array}{l}\text { Male } \\
(\mathrm{N}=29)\end{array}$} & $L L A$ & 17 & 24.9 \\
\hline & $B P$ & 4 & 27.3 \\
\hline & $S C$ & 3 & 11.0 \\
\hline & $E F$ & 5 & 23.6 \\
\hline \multirow{4}{*}{$\begin{array}{l}\text { Female } \\
(\mathrm{N}=12)\end{array}$} & $L L A$ & 0 & 24.9 \\
\hline & $B P$ & 6 & 21.8 \\
\hline & $S C$ & 3 & 15.2 \\
\hline & $E F$ & 3 & 25.7 \\
\hline
\end{tabular}

Table II: Characteristics of patient subgroups

\begin{tabular}{|l|l|c|c|}
\hline \multicolumn{1}{|c|}{ SEX } & GROUP & SUBJECTS & MEAN AGE \\
\hline Male & Patients & 29 & 23.6 \\
\cline { 2 - 4 }$(\mathrm{N}=167)$ & Controls & 138 & 30.5 \\
\hline \multirow{2}{*}{$\begin{array}{l}\text { Female } \\
(\mathrm{N}=148)\end{array}$} & Patients & 12 & 21.1 \\
\cline { 2 - 4 } & Controls & 136 & 28.0 \\
\hline
\end{tabular}

Table III: Characteristics of patient and control groups 


\title{
LEGENDS TO FIGURES
}

\section{Figure 1 Face signatures of patients; and signature weights and graphs for patients and} controls.

\begin{abstract}
A: Heat mapped face signatures of 41 patients of Dutch descent. Each demarcates localized facial dysmorphism using a red/green/blue scale for "below -2SD"/zero SD/"over +2SD" contractive/coincident/expansive surface difference compared to a mean of age-sexethnicity matched controls.
\end{abstract}

B: Face signature graph for patients (with square outline) and controls. Typically, in a signature graph the least dysmorphic individuals are located more centrally and the most dysmorphic more peripherally. The location of patient signatures largely at the periphery and terminally reflects their greater facial dysmorphism.

C. Collapsed version of the face signature graph for patients and controls, shown as empty and filled circles respectively. The annotated dispersion rates, between 0 and 1 , summarize the overall clustering of the control and patient signatures. The low value $(0.16)$ for controls and high value for patients (0.94) suggests that the patient dysmorphism is significantly different from the majority of controls. The high value for the patient group also reflects the relatively large number of clusters into which it is decomposed and the lack of homogeneity in the facial dysmorphism of its members.

D: Histogram of log-transformed normalized signature weights showing greater overall facial dysmorphism in faces of patients compared to controls. 


\section{Figure 2 Malar region definition, signature weight (for anterior-posterior depth axis)}

A: A butterfly-shaped patch of the face surface was used to demarcate a malar region. The right inner canthus $\left(I C_{R}\right)$ was connected with the right exocanthus $\left(O C_{R}\right)$ following the lower orbit border. The right exocanthus was connected with the right lower ear attachment $\left(\right.$ LEA $\left.A_{R}\right)$ and the upper ear attachment $\left(U E A_{R}\right)$ was connected with the right cheilion $\left(\mathrm{CH}_{R}\right)$; their crossing formed the lateral border. For the medial border, the right cheilion was connected with the right inner canthus following the nasolabial fold. All connecting curves followed the surface. The same procedure was repeated for the left side.

B: Histogram of log-transformed normalized signature for the malar region of patients compared to controls.

C: Signature graph for differences along the anterior-posterior axis for the malar region. Each malar signature is annotated by an outline of a different colour depending on its pattern label: BP-green; EF-black; LLA-blue; SC-red.

D: Collapsed version of signature graph shown in C using the same colour coding of pattern labels.

\section{Figure 3 Facial asymmetry signatures and associated graph for patients}

A. Heat mapped face asymmetry signatures of 41 patients of Dutch descent. Each demarcates differences of original and reflected surfaces for each patient normalized to similar differences of matched controls using a red/green/blue scale for "below -2SD"/zero SD/"over +2SD". Each heat map will necessarily be anti-symmetric in its red-blue colouring since the right sided differences of original surface face compared to its reflection or mirrored form will be the opposite for the left side. 
1

2

3

4

5

6

7

8

9

10

11

12

13

14

15

16

17

18

19

20

21

22

23

24

25

26

27

28

29

30

31

32

33

34

35

36

37

38

39

40

41

42

43

44

45

46

47

48

49

50

51

52

53

54

55

56

57

58

59

60
Hopman et al. 3D Analysis in Childhood Cancer Patients subgroup to which an individual belongs: BP-black; EF-red; LLA-green; SC-blue.

John Wiley \& Sons, Inc. 


1
2
3
4
5
6
7
8
9
10
11
12
13
14
15
16
17
18
19
20
21
22
23
24
25
26
27
28
29
30
31
32
33
34
35
36
37
38
39
40
41
42
43
44
45
46
47
48
49
50
51
52
53
54
55
56
57
58
60

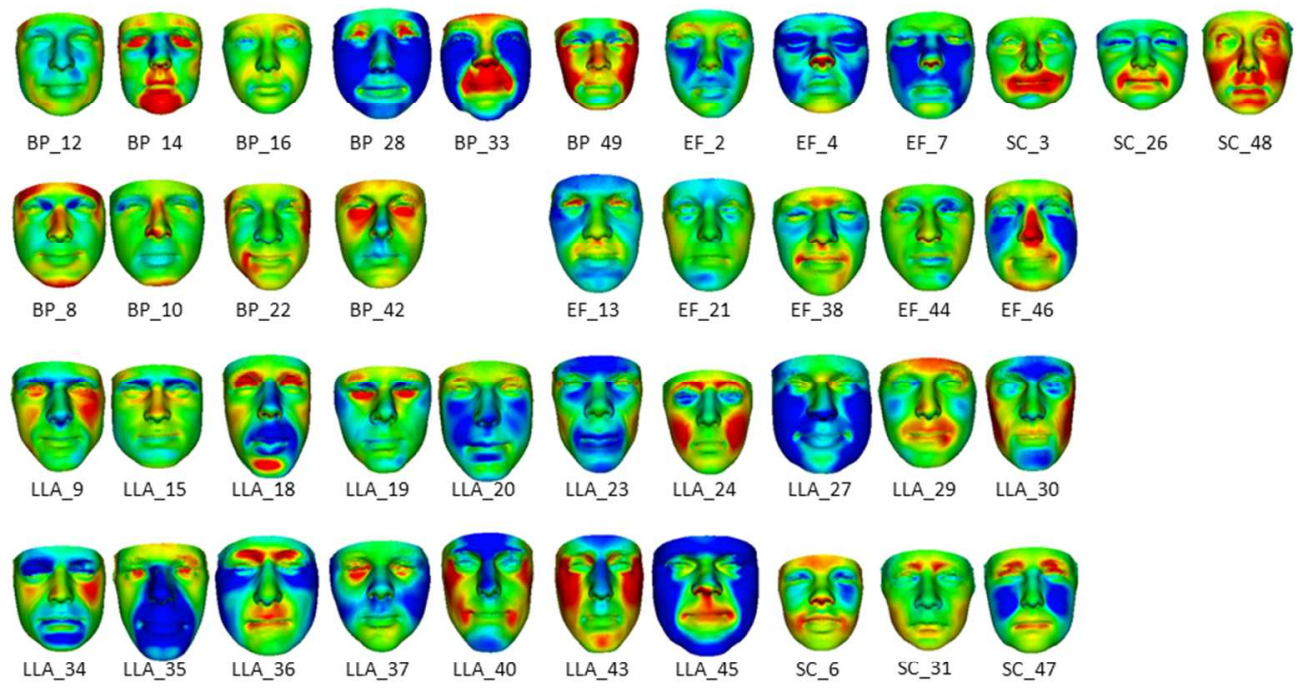

$254 \times 190 \mathrm{~mm}(96 \times 96 \mathrm{DPI})$

John Wiley \& Sons, Inc. 


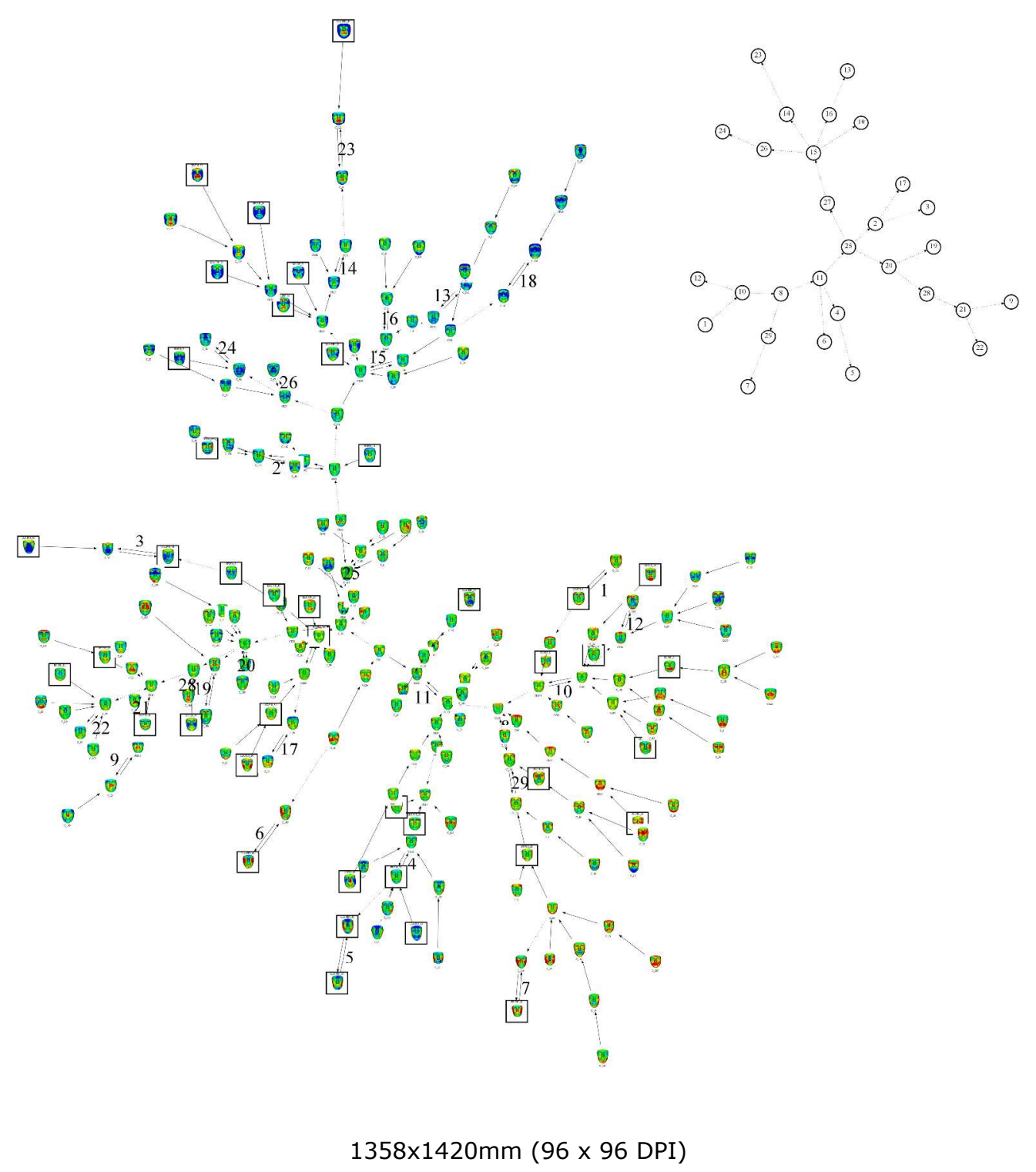

John Wiley \& Sons, Inc. 


1
2
3
4
5
6
7
8
9
10
11
12
13
14
15
16
17
18
19
20
21
22
23
24
25
26
27
28
29
30
31
32
33
34
35
36
37
38
39
40
41
42
43
44
45
46
47
48
49
50
51
52
53
54
55
56
57
58
60

-

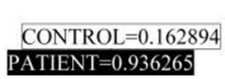

-

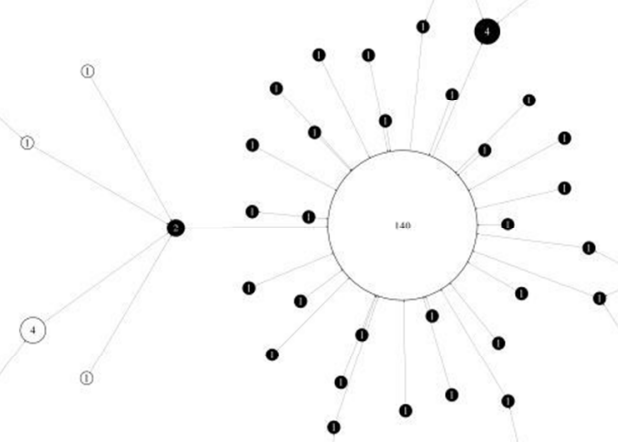

270x159mm (96 x 96 DPI)

John Wiley \& Sons, Inc. 


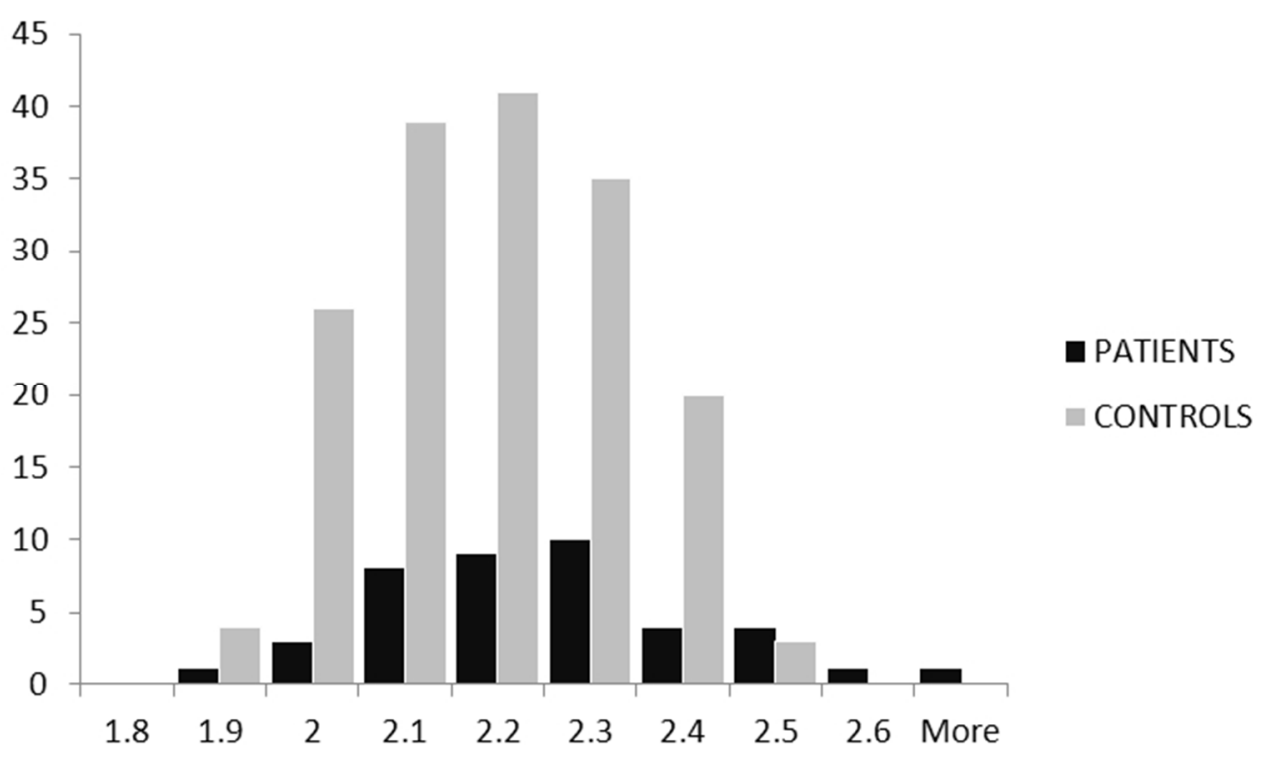

$127 \times 76 \mathrm{~mm}(150 \times 150 \mathrm{DPI})$ 


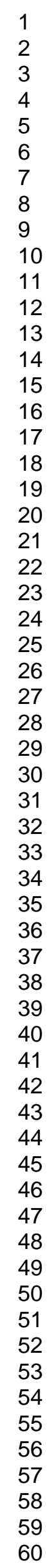

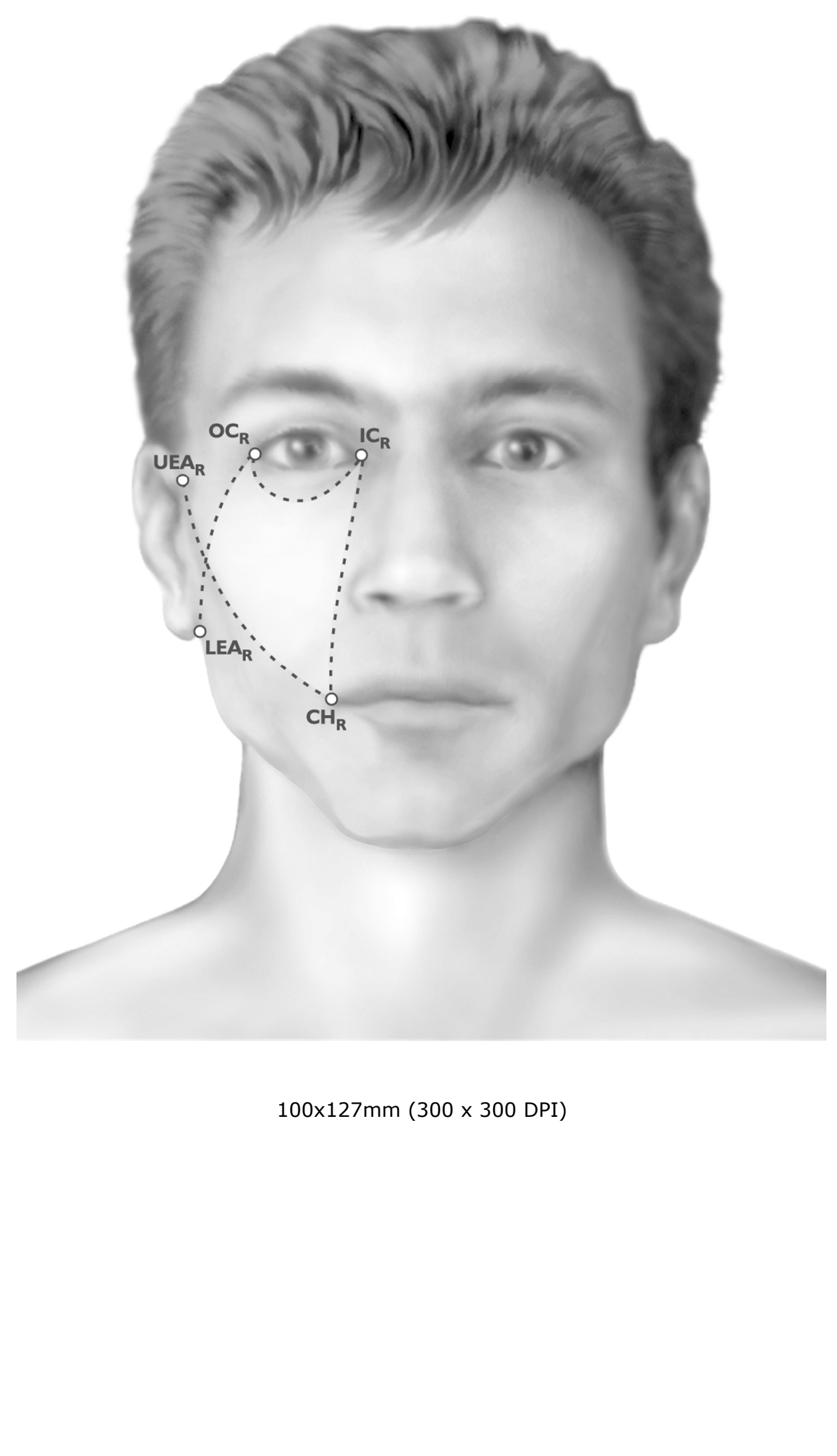

John Wiley \& Sons, Inc. 


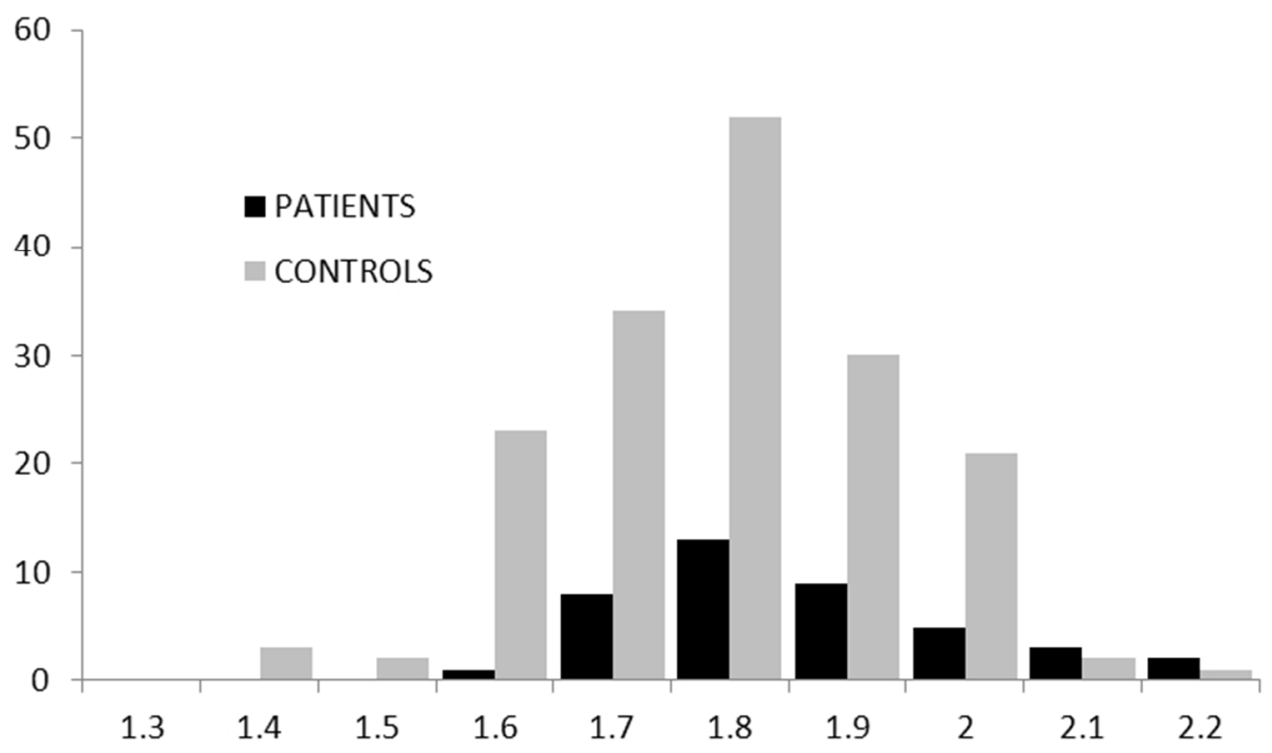

$127 \times 76 \mathrm{~mm}(150 \times 150 \mathrm{DPI})$ 


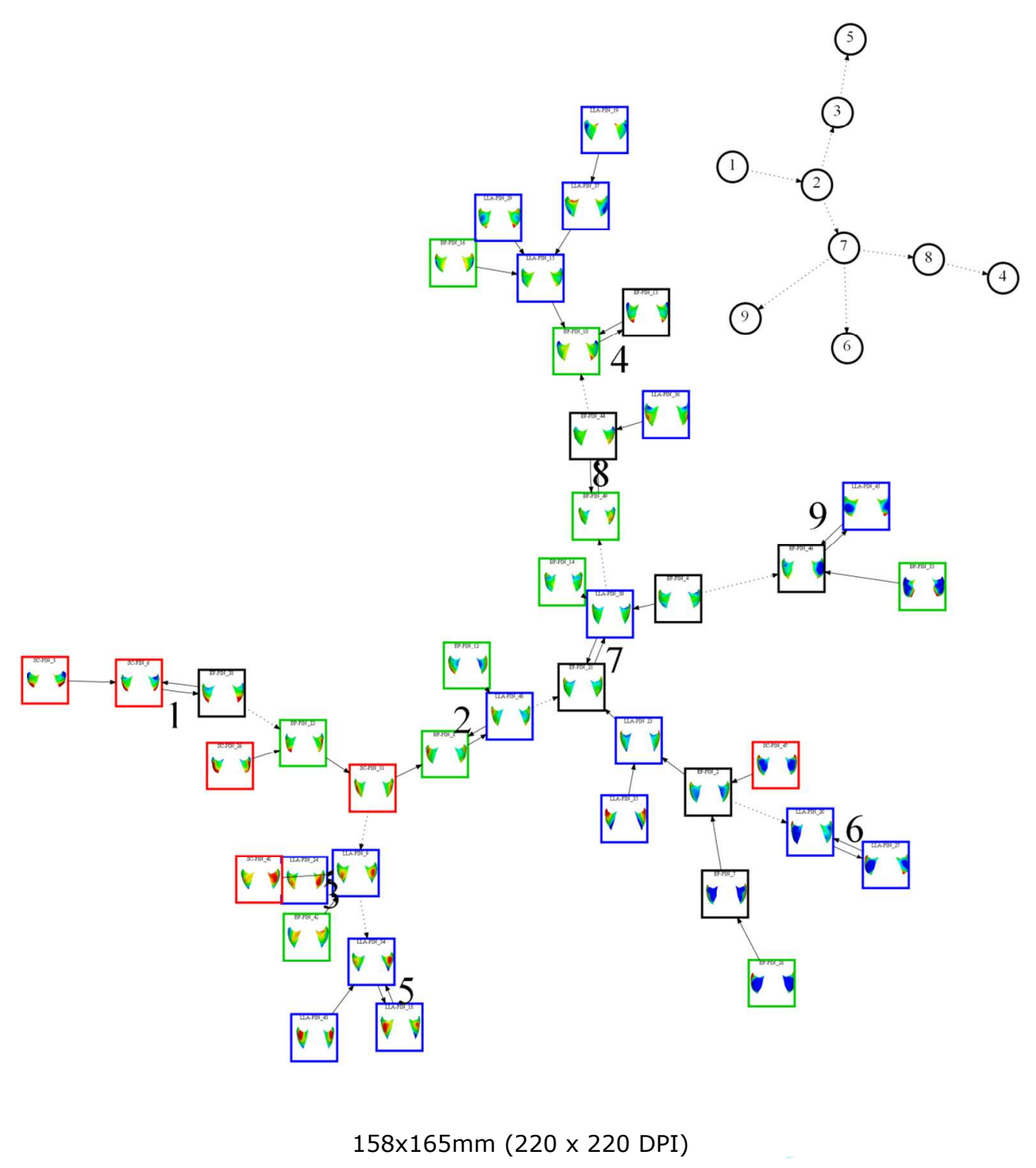

John Wiley \& Sons, Inc. 

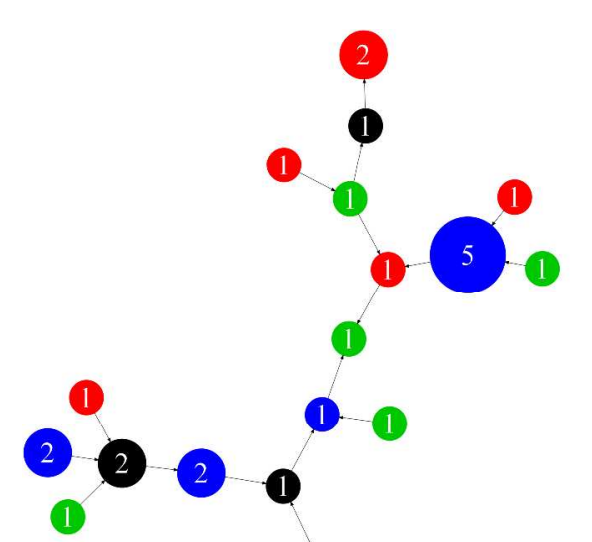

$\mathrm{LLA}=0.660229$
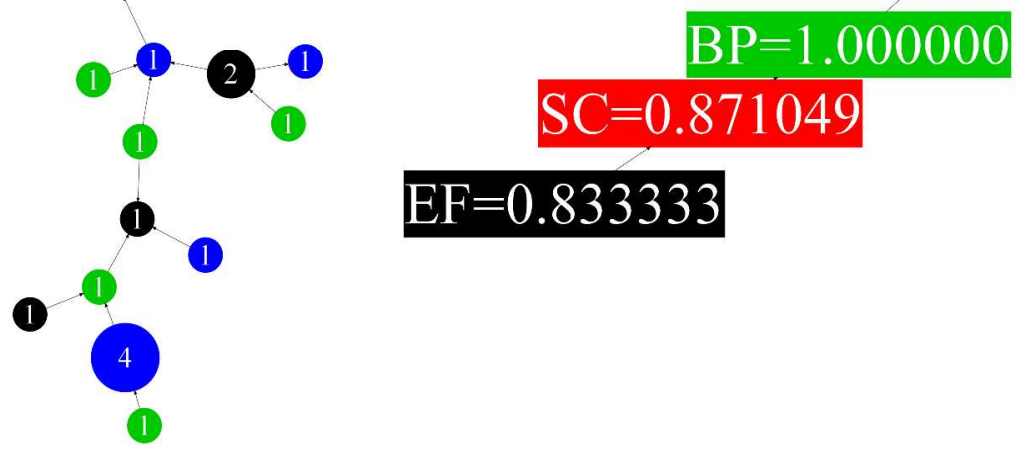

$\mathrm{EF}=0.833333$

$1133 \times 801 \mathrm{~mm}(96 \times 96 \mathrm{DPI})$

John Wiley \& Sons, Inc. 


1
2
3
4
5
6
7
8
9
10
11
12
13
14
15
16
17
18
19
20
21
22
23
24
25
26
27
28
29
30
31
32
33
34
35
36
37
38
39
40
41
42
43
44
45
46
47
48
49
50
51
52
53
54
55
56
57
58
60

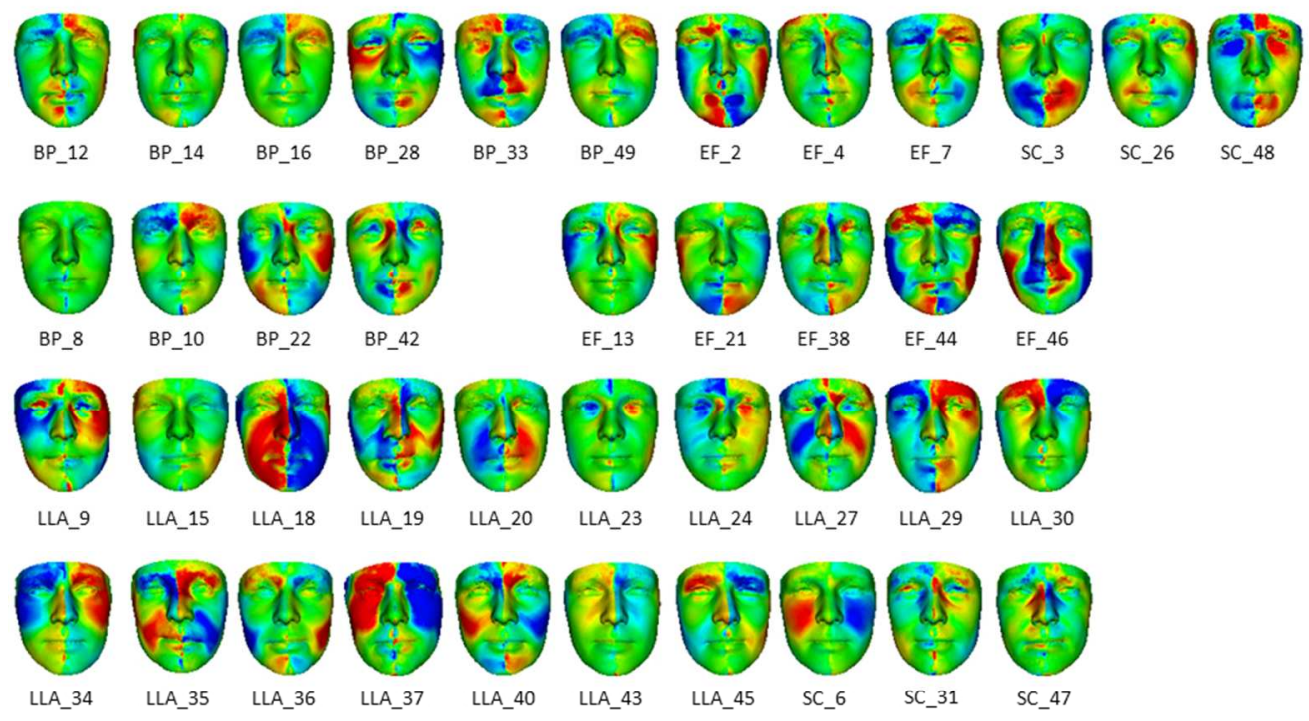

$254 \times 190 \mathrm{~mm}(96 \times 96 \mathrm{DPI})$

John Wiley \& Sons, Inc. 


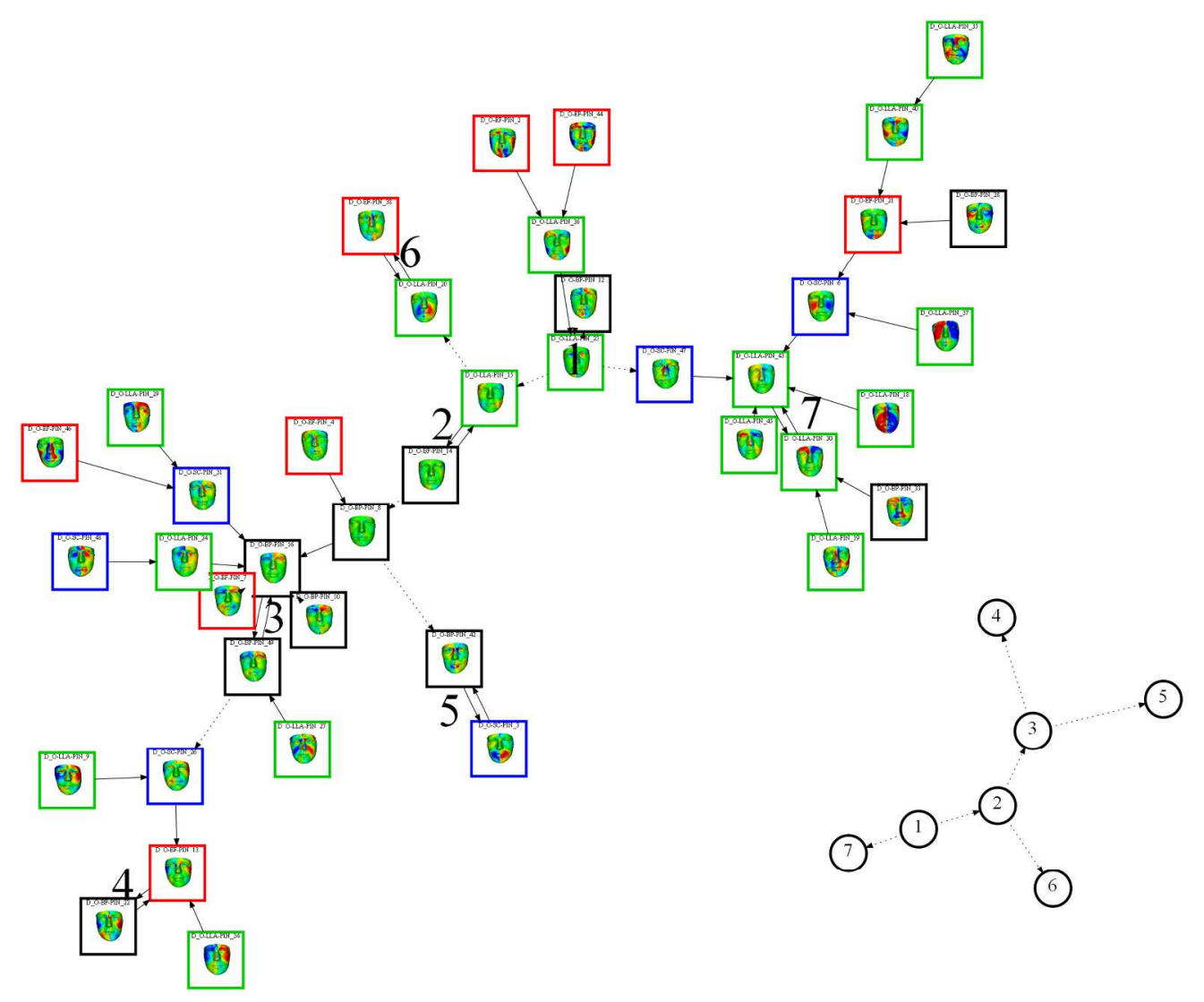

$615 \times 513 \mathrm{~mm}(96 \times 96 \mathrm{DPI})$

John Wiley \& Sons, Inc. 